\title{
Sound synthesis of a nonlinear string using Volterra series
}

\author{
Thomas Hélie ${ }^{a}$ David Roze ${ }^{a, b}$ \\ a Analysis-Synthesis team, IRCAM-CNRS UMR 9912, 1 place Igor Stravinsky, \\ 75004 Paris, France \\ ${ }^{\mathrm{b}}$ CEA-LIST, Route du panorama, 92265 Fontenay-aux-roses CEDEX, France
}

\begin{abstract}
This paper proposes to solve and simulate various Kirchhoff models of nonlinear strings using Volterra series. Two nonlinearities are studied: the string tension is supposed to depend either on the global elongation of the string (first model), or on the local strain located at $x$ (second, and more precise, model). The boundary conditions are simple Dirichlet homogeneous ones or general dynamic conditions (allowing the string to be connected to any system; typically a bridge). For each model, a Volterra series is used to represent the displacement as a functional of excitation forces. The Volterra kernels are solved using a modal decomposition: the first kernel of the series yields the standard modes of the linearized problem while the next kernels introduce the nonlinear dynamics. As a last step, systematic identification of the kernels lead to a structure composed of linear filters, sums, and products which are well-suited to the sound synthesis, using standard signal processing techniques. The nonlinear dynamic introduced through this simulation is significant and perceptible in sound results for sufficiently large excitations.
\end{abstract}

Key words: string, nonlinear model, Volterra series, analytic solution, modal decomposition, sound synthesis.

\section{Introduction}

In musical acoustics, sound synthesis aims to produce a more and more realistic result for complex systems such as musical instruments. Usually, these instruments involve nonlinear propagation phenomena as soon as vibrations

Email addresses: thomas.helie@ircam.fr (Thomas Hélie), david.roze@ircam.fr (David Roze).

Preprint submitted to Elsevier 2 April 2008 
are sufficiently large in instruments such as gongs, dynamics of bowed strings, piano soundboards, etc. Thus, physical models which include nonlinear phenomena have been derived and usually solved using numerical methods, as in [1]. For sinusoidal type excitations, methods such as nonlinear modes $[2,3]$ or the harmonic balance $[4,5]$ are interesting alternatives.

In this article, nonlinear models of damped strings are solved using Volterra series (see $[6,7]$ ). This method allows both analytic and numerical solutions to be performed without requiring sinusoidal-type excitations. More precisely, once the analytic kernels of the series are derived, straightforward identifications lead to structures composed of linear filters, sums, and products. This yields efficient simulations from standard signal processing techniques, which are well-adapted to real-time synthesis. Moreover, decomposing the kernels on the modal basis yields original but natural interpretations: it exhibits precisely the nonlinear dynamics of each spatial mode.

Physical models of strings are numerous. Historically, the first information on the equations of the motion of a string appeared in the 18th century with the works of d'Alembert [8] and Euler [9] who respectively wrote the two linear partial differential equations for small vibrations of a string and transverse vibrations of a bar (see also [10]). During the 19th century, Kirchhoff derived a model of a one-dimensional perfectly flexible string, including a nonlinearity due to the variation of tension [11]. This model has been re-investigated by Carrier in [12] from which new string models of musical instruments have been elaborated. For instance, in [13], Anand focused on the non-planar transverse vibrations (with celerity $c_{l}$ ) and neglected the longitudinal ones (with celerity $c_{l}$ ), assuming that $c_{l}^{2} \gg c_{t}^{2}$. On the other hand, in [14], Narasimha states that the longitudinal motion cannot be neglected even for small amplitudes. Both these works (and others) have been unified by A. Watzky in [15] with a three-dimensional model of a nonlinear stiff string. This generalization includes a torsion coupling and allows the introduction of inharmonicity using the hypothesis of a linear elastic behaviour. Advanced models and experimental results on strings can also be found in [16].

This work focuses on three mono-dimensional nonlinear models (M1,M2,M3) of perfectly flexible strings with large transverse waves: the nonlinearity can be globally integrated (M1,M2) or locally distributed (M3) in space; the boundary conditions can be homogeneous (M1,M3) or it is possible to connect the string to another system, typically, a bridge (M2).

The paper is organized as follows: Section 2 presents the three models; Section 3 introduces the Volterra series; Sections 4 to 6 detail the calculation of the Volterra kernels for (M1) to (M3), from which simulable structures are deduced. Section 7 presents results and comparisons in both the time and time-frequency domains. Section 8 develops conclusions and perspectives. 


\section{Physical models of strings}

\subsection{Geometry, physical constants, and excitation}

Consider the displacement $u(x, t)$ of a perfectly flexible string (Fig. 1) with a length $L[\mathrm{~m}]$, an initial tension $T_{0}[\mathrm{~N}]$, and a small circular section with radius $R[\mathrm{~m}]$. The material is characterized by a density $\rho\left[\mathrm{Kg} \mathrm{m}^{-3}\right]$, a Young's modulus $E[\mathrm{~Pa}]$, a standard fluid damping $\delta\left[\mathrm{s}^{-1}\right]$ introduced by the transverse mass force $-\delta \frac{\partial u}{\partial t}$, and a structural damping $\kappa\left[\mathrm{m}^{2} . \mathrm{s}^{-1}\right]$ introduced by $+\kappa \frac{\partial^{3} u}{\partial t \partial x^{2}}$. These dampings model thermoelastic losses and viscoelasticity [17]. The string

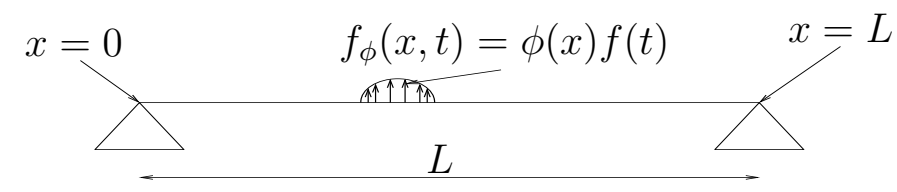

Fig. 1. Characteristics of the string

is supposed to be motionless before $t=0$ and the transverse displacement is denoted $u(x, t)$ for all $(x, t) \in \bar{\Omega} \times \mathbb{R}^{+}$with $\left.\Omega=\right] 0, L[$ and $\bar{\Omega}=[0, L]$. It is excited by a transverse mass force $f_{\phi}(x, t)=\phi(x) f(t)$ where $\phi$ is positive and spatially distributes the total force $f(t)$, so that

$$
\rho A \int_{\Omega} \phi(x) \mathrm{d} x=1 \quad \text { with } A=\pi R^{2} .
$$

Two simplified models of the nonlinear propagation (NL1,NL2) and two kinds of boundary conditions $(\mathrm{B} 1, \mathrm{~B} 2)$ are described below for transverse vibrations.

\subsection{Nonlinear models of propagation, boundary and initial conditions}

\subsubsection{Model with a global variation of tension (NL1)}

The Kirchhoff equation models the transverse vibrations of a string [11]. If the fluid and structural damping forces are included, it is given by

$$
\forall(x, t) \in \Omega \times \mathbb{R}_{+}^{*}, \frac{\partial^{2} u}{\partial t^{2}}+\delta \frac{\partial u}{\partial t}-\kappa \frac{\partial^{3} u}{\partial t \partial x^{2}}=\left[c^{2}+b \int_{0}^{L}\left(\frac{\partial u}{\partial x}\right)^{2} \mathrm{~d} x\right] \frac{\partial^{2} u}{\partial x^{2}}+f_{\phi},
$$

where $c=\sqrt{\frac{T_{0}}{\rho A}}$ is the wave celerity $\left[\mathrm{m} . \mathrm{s}^{-1}\right]$ and $b=\frac{E}{2 \rho L}$ is the coefficient of the nonlinearity $\left[\mathrm{m} . \mathrm{s}^{-2}\right]$ which takes into account the variation of tension. This model holds under three hypotheses [18]: (H1) the transverse vibrations are confined to a plane; (H2) the string is perfectly flexible (second order equation); (H3) the nonlinear effects are due to the global variation of length. 


\subsubsection{Model with a local variation of tension (NL2)}

The Kirchhoff-Carrier equations model both the longitudinal $(v)$ and the transverse $(u)$ vibrations which are coupled through the variation of tension. For an undamped string [12], they are given by, $\forall(x, t) \in \Omega \times \mathbb{R}_{+}^{\star}$,

$$
\rho A \frac{\partial^{2} u}{\partial t^{2}}=\frac{\partial}{\partial x}\left[\frac{T \frac{\partial u}{\partial x}}{\sqrt{\left(\frac{\partial u}{\partial x}\right)^{2}+\left(1+\frac{\partial v}{\partial x}\right)^{2}}}\right] \text { and } \rho A \frac{\partial^{2} v}{\partial t^{2}}=\frac{\partial}{\partial x}\left[\frac{T\left(1+\frac{\partial v}{\partial x}\right)}{\sqrt{\left(\frac{\partial u}{\partial x}\right)^{2}+\left(1+\frac{\partial v}{\partial x}\right)^{2}}}\right]
$$

where $T-T_{0}=E A\left(\sqrt{\left(\frac{\partial u}{\partial x}\right)^{2}+\left(1+\frac{\partial v}{\partial x}\right)^{2}}-1\right)$. Neglecting $\frac{\partial v}{\partial x}$ in Eq. (3a) and including the fluid and structural damping forces define the model (NL2) which governs a damped wave $u$ decoupled from $v$ :

$$
\rho A \frac{\partial^{2} u}{\partial t^{2}}+\rho A \delta \frac{\partial u}{\partial t}-\rho A \kappa \frac{\partial^{3} u}{\partial t \partial x^{2}}=\frac{\partial}{\partial x}\left[\frac{\left(T_{0}+E A\left(\sqrt{1+\left(\frac{\partial u}{\partial x}\right)^{2}}-1\right)\right) \frac{\partial u}{\partial x}}{\sqrt{1+\left(\frac{\partial u}{\partial x}\right)^{2}}}\right] .
$$

Note that this model involves a nonlinearity (local with respect to $x$ ) with no integral operator as in (NL1). In this model, (H3) is relaxed, but only transverse waves are (still) assumed to be significant.

This model (NL2) will illustrate how the solutions and the simulations are modified because of local-rather than global-nonlinearities. A more realistic model with coupled waves will be solved in a future work.

\subsubsection{Boundary conditions (B1,B2) and initial conditions}

Boundary conditions (B1) are homogeneous Dirichlet conditions, i.e. the string has no displacement at extremities:

$$
\forall(x, t) \in\{0 ; L\} \times \mathbb{R}^{+}, \quad u(x, t)=0 .
$$

Boundary conditions (B2) are defined by

$$
\forall(x, t) \in\{0 ; L\} \times \mathbb{R}^{+}, \quad u(x, t)=u_{x}(t),
$$

where $u_{0}$ and $u_{L}$ represent excitations. These excitations are both displacements but could be both forces $\left(\frac{\partial u}{\partial x}(x, t)=u_{x}(t)\right.$ for $\left.(x, t) \in\{0 ; L\} \times \mathbb{R}^{+}\right)$, or even mixed excitations. These choices do not affect the method proposed in the following, but only the solutions. The latter conditions (B2) allow the string to be connected to a bridge or to other mechanical systems, at its extremities. 
For all the cases studied here, the string is supposed to be motionless for $t \leq 0$ so that initial conditions are zero:

$$
\forall(x, t) \in \Omega \times\{0\}, k \in\{0 ; 1\}, \quad \frac{\partial^{k} u}{\partial t^{k}}(x, t)=0 .
$$

\subsection{Dimensionless models}

The changes of variables and functions given in Table 1 yield the dimensionless models $(\mathrm{M} 1, \mathrm{M} 2, \mathrm{M} 3)$ on $\widetilde{\Omega}=] 0,1[$ :

$(\mathrm{M} 1) \equiv(\mathrm{NL1}, \mathrm{B1})$ Clamped string with a global variation of tension:

$$
\begin{aligned}
& \forall(\tilde{x}, \tilde{t}) \in \tilde{\Omega} \times \mathbb{R}_{+}^{\star}, \quad \frac{\partial^{2} \tilde{u}}{\partial \tilde{t}^{2}}+\alpha \frac{\partial \tilde{u}}{\partial \tilde{t}}-\beta \frac{\partial^{3} \tilde{u}}{\partial \tilde{t} \partial \tilde{x}^{2}}=\left[1+\epsilon \int_{0}^{1}\left(\frac{\partial \tilde{u}}{\partial \tilde{x}}\right)^{2} \mathrm{~d} \tilde{x}\right] \frac{\partial^{2} \tilde{u}}{\partial \tilde{x}^{2}}+\tilde{f}_{\tilde{\phi}}, \\
& \forall(\tilde{x}, \tilde{t}) \in\{0 ; 1\} \times \mathbb{R}_{+}^{\star}, \tilde{u}(\tilde{x}, \tilde{t})=0 \\
& \forall(\tilde{x}, \tilde{t}) \in \tilde{\Omega} \times\{0\}, \quad \tilde{u}(\tilde{x}, \tilde{t})=0 \text { and } \frac{\partial \tilde{u}}{\partial \tilde{t}}(\tilde{x}, \tilde{t})=0
\end{aligned}
$$

$(\mathrm{M} 2) \equiv(\mathrm{NL1}, \mathrm{B2})$ Same string with dynamic boundary conditions:

$$
\begin{aligned}
& \forall(\tilde{x}, \tilde{t}) \in \tilde{\Omega} \times \mathbb{R}_{+}^{\star}, \quad \frac{\partial^{2} \tilde{u}}{\partial \tilde{t}^{2}}+\alpha \frac{\partial \tilde{u}}{\partial \tilde{t}}-\beta \frac{\partial^{3} \tilde{u}}{\partial \tilde{t} \partial \tilde{x}^{2}}=\left[1+\epsilon \int_{0}^{1}\left(\frac{\partial \tilde{u}}{\partial \tilde{x}}\right)^{2} \mathrm{~d} \tilde{x}\right] \frac{\partial^{2} \tilde{u}}{\partial \tilde{x}^{2}}+\tilde{f}_{\tilde{\phi}},(11 \\
& \forall(\tilde{x}, \tilde{t}) \in\{0 ; 1\} \times \mathbb{R}_{+}^{\star}, \tilde{u}(\tilde{x}, \tilde{t})=\tilde{u}_{\tilde{x}}(\tilde{t}) \\
& \forall(\tilde{x}, \tilde{t}) \in \tilde{\Omega} \times\{0\}, \quad \tilde{u}(\tilde{x}, \tilde{t})=0 \text { and } \frac{\partial \tilde{u}}{\partial \tilde{t}}(\tilde{x}, \tilde{t})=0
\end{aligned}
$$

(M3) $\equiv(\mathrm{NL2,B1)}$ Clamped string with local variations of tension:

$$
\begin{aligned}
& \forall(\tilde{x}, \tilde{t}) \in \tilde{\Omega} \times \mathbb{R}_{+}^{\star}, \quad \frac{\partial^{2} \tilde{u}}{\partial \tilde{t}^{2}}+\alpha \frac{\partial \tilde{u}}{\partial \tilde{t}}-\beta \frac{\partial^{3} \tilde{u}}{\partial \tilde{t} \partial \tilde{x}^{2}}=\frac{\partial}{\partial \tilde{x}}\left[\frac{\left(1-\frac{2 \epsilon}{\eta}\right) \frac{\partial \tilde{u}}{\partial \tilde{x}}}{\sqrt{1+\eta\left(\frac{\partial \tilde{u}}{\partial \tilde{x}}\right)^{2}}}+\frac{2 \epsilon}{\eta} \frac{\partial \tilde{u}}{\partial \tilde{x}}\right]+\tilde{f}_{\tilde{\phi}},(14 \\
& \forall(\tilde{x}, \tilde{t}) \in\{0 ; 1\} \times \mathbb{R}_{+}^{\star} \tilde{u}(\tilde{x}, \tilde{t})=0 \\
& \forall(\tilde{x}, \tilde{t}) \in \tilde{\Omega} \times\{0\}, \quad \tilde{u}(\tilde{x}, \tilde{t})=0 \text { and } \frac{\partial \tilde{u}}{\partial \tilde{t}}(\tilde{x}, \tilde{t})=0
\end{aligned}
$$

For sake of legibility, the tilde symbols will be omitted in the following.

\section{Introduction to Volterra series}

Volterra series have been mainly used to solve nonlinear electronic circuits and ordinary differential equations including regular nonlinearities (see [2124]). They represent the solution (for "control engineers", the output of a system) as an infinite sum of multi-convolutions fed by the excitation (the 


\begin{tabular}{|l|l||l|l|}
\hline \multicolumn{2}{|c||}{ Dimensional } & \multicolumn{2}{c|}{ Dimensionless } \\
\hline geometry & physical constants & variables / coefficients & functions \\
\hline \hline \multirow{2}{*}{$L=1.8 \mathrm{~m}$} & $\rho=7800 \mathrm{Kg} \mathrm{m}{ }^{-3}$ & $\tilde{x}=\frac{x}{L}, \quad \tilde{t}=\frac{t c}{L}$ & $\tilde{u}(\tilde{x}, \tilde{t})=\frac{u\left(\tilde{x} L, \frac{\tilde{t} L}{c}\right)}{U^{\star}}$ \\
$R=1.5 \mathrm{~mm}$ & $E=210^{11} \mathrm{~Pa}$ & $\alpha=\frac{\delta L}{c}=5.4510^{-2}$ & $\tilde{\phi}(\tilde{x})=\rho \pi R^{2} L \phi(\tilde{x} L)$ \\
\cline { 1 - 1 } Reference & $\delta=3 \mathrm{~s}^{-1}$ & $\beta=\frac{\kappa}{c L}=2.8110^{-5}$ & $\tilde{f}(\tilde{t})=\frac{L f\left(\frac{\tilde{t} L}{c}\right)}{\rho \pi R^{2} c^{2} U^{\star}}$ \\
displacement: & $\kappa=0.01 \mathrm{~m}^{2} \mathrm{~s}^{-1}$ & $\epsilon=\frac{E\left(U^{\star}\right)^{2}}{2 \rho(L c)^{2}}=2.2710^{-4}$ & $\tilde{f}_{\tilde{\phi}}(\tilde{x}, \tilde{t})=\tilde{\phi}(\tilde{x}) \tilde{f}(\tilde{t})$ \\
$U^{\star}=R$ & $T_{0}=2161 \mathrm{~N}$ & $\eta=\left(\frac{U^{\star}}{L}\right)^{2}=6.9410^{-7}$ & $\tilde{u}_{\tilde{x}}(\tilde{t})=\frac{u_{\tilde{x} L}(\tilde{t} L)}{U^{\star}}$ \\
\hline
\end{tabular}

Table 1

Changes of variables, of functions, and of dimensionless coefficients (Eq. (1) translates into $\left.\int_{0}^{1} \tilde{\phi}(\tilde{x}) \mathrm{d} \tilde{x}=1\right)$. The proposed values are used in $\S 7$ : the string is made of steel[19,20]; $\delta, \kappa$, and $T_{0}$ are tuned to have realistic damped sounds and the fundamental frequency $f=\frac{c}{2 L}=55 \mathrm{~Hz}$ with $c=\sqrt{\frac{T_{0}}{\rho A}}$.

input of a system). It extends "linear filtering" to the case of "weakly nonlinear behaviours".

\subsection{Definition and properties}

A causal system (in the "control engineers' meaning") with input $f$, output $u$ (see Fig. 2) is described by a Volterra series with kernels $\left\{h_{n}\right\}_{n \in \mathbb{N}^{*}}$ if

$$
\forall t \in \mathbb{R}^{+}, \quad u(t)=\sum_{n=1}^{\infty} \int_{\left(\mathbb{R}^{+}\right)^{n}} h_{n}\left(\tau_{1: n}\right) f\left(t-\tau_{1}\right) \ldots f\left(t-\tau_{n}\right) \mathrm{d} \tau_{1: n}
$$

with the notation $\left(\tau_{1: n}\right)=\left(\tau_{1}, \tau_{2}, \ldots, \tau_{n}\right)$ and $\mathrm{d} \tau_{1: n}=\mathrm{d} \tau_{1} \mathrm{~d} \tau_{2} \ldots \mathrm{d} \tau_{n}$.

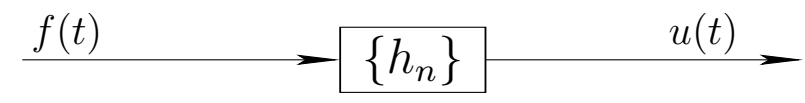

Fig. 2. System with input $f$ and output $u$ described by a Volterra series $\left\{h_{n}\right\}_{n \in \mathbb{N}^{*}}$.

Eq. (17) can be interpreted as follows: for $n=1$, the term is a standard linear convolution so that $h_{1}$ is the impulse response of the linear contribution. For $n=2$, the term introduces a quadratic contribution of $f$ on the output $u$. More generally, the $n$-th term is associated with a homogeneous nonlinearity of order $n$ which takes into account some memory through a "multi-convolution".

Moreover, as in the linear case, generalized transfer functions $H_{n}\left(s_{1: n}\right)$ (denoted with capital letters) can be defined as the Laplace transform of the generalized impulse responses $h_{n}\left(\tau_{1: n}\right)\left(\left(s_{1: n}\right)=\left(s_{1}, \ldots, s_{n}\right)\right.$ denotes the Laplace 
variables). For a damped (i.e. stable) causal system, they are defined by

$$
\forall\left(s_{1: n}\right) \in \mathcal{D} \supset\left(\mathbb{C}_{0}^{+}\right)^{n}, \quad H_{n}\left(s_{1: n}\right)=\int_{\left(\mathbb{R}^{+}\right)^{n}} h_{n}\left(\tau_{1: n}\right) \mathrm{e}^{-\left(s_{1} \tau_{1}+\ldots+s_{n} \tau_{n}\right)} \mathrm{d} \tau_{1: n}
$$

where $\mathcal{D}$ is the domain of definition and $\mathbb{C}_{0}^{+}$is the set of complex numbers with a strictly positive real part (see [25, (29.1.2)] and [22] for more details). Remark 1: Embedded Volterra series are: (a) linear filters $\left(h_{n}=0\right.$ for $\left.n \geq 2\right)$; (b) instantaneous functions $u(t)=h(f(t))$ which admits a series expansion $h(f)=\sum_{n=1}^{+\infty} \alpha_{n} f^{n}$; (c) their combinations (sum, product, cascade, see $\S 3.2$ ).

Remark 2: For the case (b), $h_{n}\left(\tau_{1: n}\right)=\alpha_{n} \delta\left(\tau_{1: n}\right)$ in the time domain ( $\delta$ denotes the Dirac distribution), and $H_{n}\left(s_{1: n}\right)=\alpha_{n}$ in the Laplace domain.

Remark 3: For inputs $f$ which are zero before $t=0$, Eq. (17) becomes $u(t)=\sum_{n=1}^{\infty} \int_{[0, t]^{n}} h_{n}\left(\tau_{1: n}\right) f\left(t-\tau_{1}\right) \ldots f\left(t-\tau_{n}\right) \mathrm{d} \tau_{1: n}$. Such a dynamic is that of a system with null initial conditions. This limitation can be removed using a more general definition of Volterra series (see [24]).

Remark 4: From a mathematical point of view, the convergence of the series holds for inputs $f$ such that $|f(t)|<\rho$ where $\rho$ is the convergence radius of the characteristic function $\varphi_{h}(x)=\sum_{n=1}^{+\infty}\left\|h_{n}\right\|_{1} x^{n}$ with the $L^{1}$-norm $\left\|h_{n}\right\|_{1}=\int_{\mathbb{R}^{n}}\left|h_{n}\left(\tau_{1: n}\right)\right| \mathrm{d} \tau_{1: n}$ (see $\left.[22]\right)$. In this case, $|u(t)| \leq \phi_{h}\left(\sup _{t \in \mathbb{R}}|f(t)|\right)$ (see $[24,26,27]$ for other results on the convergence). Nevertheless, we will not study the convergence of the Volterra series in this paper: in practice, low order truncations of the series yield good approximations, if the nonlinearity is not activated by "too large inputs", as discussed in $\S 7$.

\subsection{Interconnection laws}

The following interconnection laws will be useful to solve (M1)-(M3).

Consider two Volterra series with kernels $\left\{a_{n}\right\}_{n \in \mathbb{N}^{*}}$ and $\left\{b_{n}\right\}_{n \in \mathbb{N}^{*}}$. The systems with input $f$ and output $u$ defined respectively in Fig. 3, in Fig. 4 and, if $b_{n}=0$ for $n \geq 2$, in Fig. 5 are still described by a Volterra series. Their kernels $\left\{c_{n}\right\}_{n \in \mathbb{N}^{*}}$ are given in the Laplace domain by, respectively [22, p. 34,35],

$$
\begin{aligned}
& C_{n}\left(s_{1: n}\right)=A_{n}\left(s_{1: n}\right)+B_{n}\left(s_{1: n}\right) \\
& C_{n}\left(s_{1: n}\right)=\sum_{p=1}^{n-1} A_{p}\left(s_{1: p}\right) B_{n-p}\left(s_{p+1: n}\right) \\
& C_{n}\left(s_{1: n}\right)=A_{n}\left(s_{1: n}\right) B_{1}\left(\widehat{s_{1: n}}\right)
\end{aligned}
$$

with the notation $\widehat{s_{1: n}}=s_{1}+\ldots+s_{n}$. 


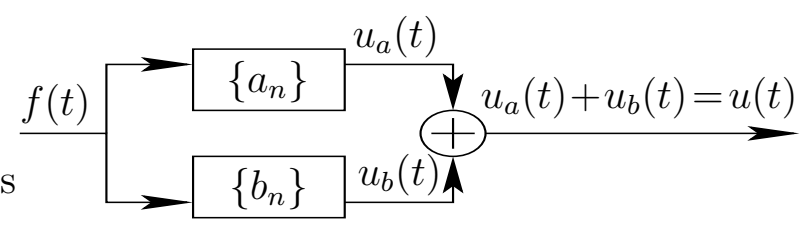

Fig. 3. Interconnection: sum of outputs.

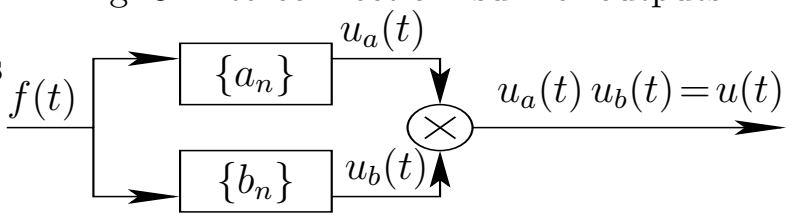

Fig. 4. Interconnection: product of outputs.

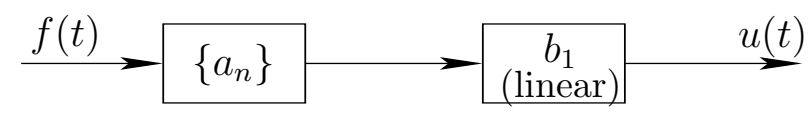

Fig. 5. Interconnection: cascade of a Volterra system with a linear system.

\section{Solution and simulation of (M1) using a Volterra series}

In this section, the displacement $u(x, t)$ of a string governed by (M1) and driven by a force $f$ starting from $t=0$ is solved. The spatial distribution $\phi$ is considered as an a priori given data of the problem. In $\S 4.1$, the Volterra series which maps $f$ to $u$ is defined. In $\S 4.2$, the kernels of the series are solved and explicit expressions are given. In $\S 4.3$, structures composed for linear filters, sums and products are identified from which a numerical simulation is derived.

\subsection{Derivation of the Volterra kernels for (M1)}

\subsubsection{Modelling the solution by a Volterra series.}

Consider the displacement $u(x, t)$ as the output of a system (in the "control engineers' meaning") with input $f(t)$. This defines a nonlinear system that depends on the space variable $x$ so that the corresponding Volterra series has kernels parameterized by $x$, denoted $h_{n}^{(x)}$ (see Fig. 6). The solution is represented by

$$
\forall(x, t) \in \bar{\Omega} \times \mathbb{R}^{+}, \quad u(x, t)=\sum_{n=1}^{\infty} \int_{[0, t]^{n}} h_{n}^{(x)}\left(\tau_{1: n}\right) f\left(t-\tau_{1}\right) \ldots f\left(t-\tau_{n}\right) \mathrm{d} \tau_{1: n}
$$

Note that, from remark 3 in $§ 3.1$, this solution naturally satisfies Eq. (10).

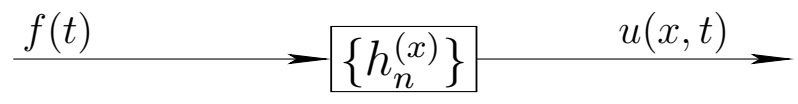

Fig. 6. Volterra system representing the solution $u(x, t)$ of (M1). 


\subsubsection{Equation satisfied by the kernels inside the domain $\Omega$.}

Eq. (8) requires that the kernels $h_{n}^{(x)}$ satisfy an equation inside $\Omega$. This equation can be derived in the Laplace domain using the interconnection laws (see $\S 3.2$ ), as detailed below.

The block-diagram in Fig. 7@ translates Eq. (8) into $w_{a}(x, t)+w_{b}(x, t) w_{c}(x, t)+$ $w_{d}(x, t)=0$, where $w_{a}=\frac{\partial^{2} u}{\partial t^{2}}+\alpha \frac{\partial u}{\partial t}-\left(1+\beta \frac{\partial}{\partial t}\right) \frac{\partial^{2} u}{\partial x^{2}}, w_{b}=-\epsilon \int_{0}^{1}\left(\frac{\partial u}{\partial x}\right)^{2} \mathrm{~d} x$, $w_{c}=\frac{\partial^{2} u}{\partial x^{2}}, w_{d}(x, t)=\phi(x) f(t)$, and where $u$ is represented by Eq. (21). Each

(a)

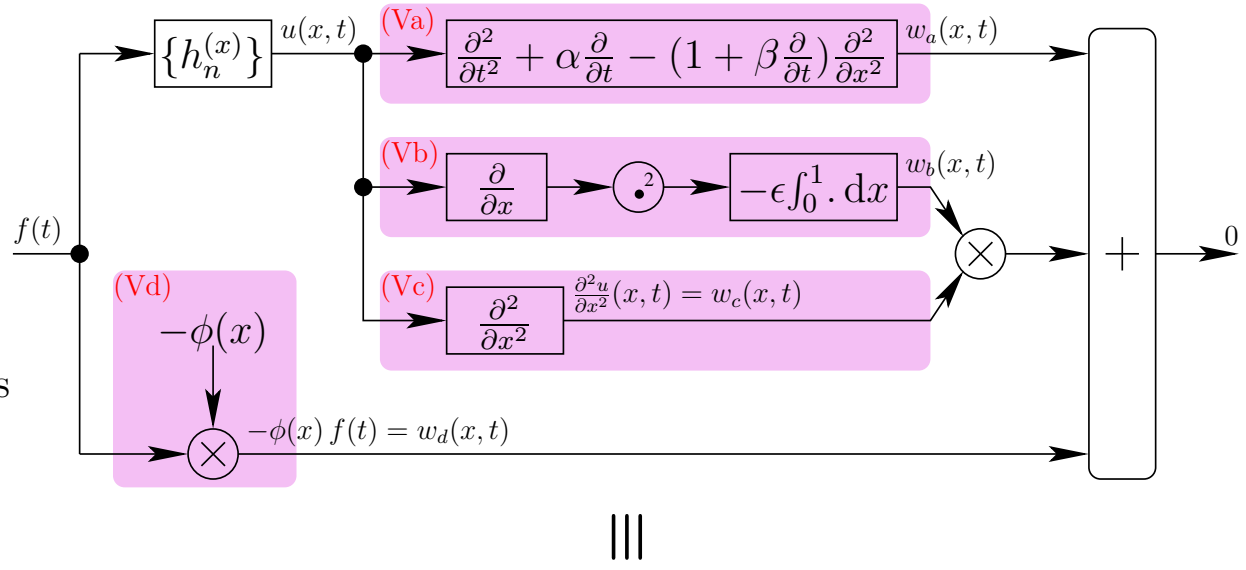

(b)

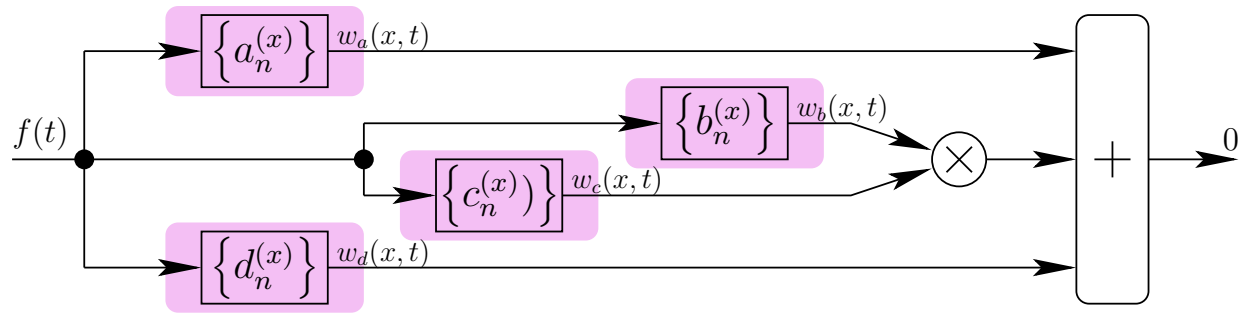

Fig. 7. Equivalent block-diagrams representing Eq. (8).

system which maps $f$ to $w_{\{a, b, c, d\}}$ can be represented by a Volterra series (see Fig. 7(b)), the kernels of which can be deduced from interconnection laws, in the Laplace domain, as follows:

Block (Va): in the Laplace domain (with respect to the time variable), the operator $\frac{\partial^{2}}{\partial t^{2}}+\alpha \frac{\partial}{\partial t}-\left(1+\beta \frac{\partial}{\partial t}\right) \frac{\partial^{2}}{\partial x^{2}}$ becomes $s^{2}+\alpha s-(1+\beta s) \frac{\partial^{2}}{\partial x^{2}}$. Thus, from Eq. (20), the cascade of $\left\{H_{n}^{(x)}\right\}$ and this linear operator defines the Volterra series $\left\{A_{n}^{(x)}\right\}$ (see Fig.7(b)) with, for all $n \in \mathbb{N}^{*}$,

$$
A_{n}^{(x)}\left(s_{1: n}\right)=\left[\left(\widehat{s_{1: n}}\right)^{2}+\alpha \widehat{s_{1: n}}-\left(1+\beta \widehat{s_{1: n}}\right) \frac{\partial^{2}}{\partial x^{2}}\right] H_{n}^{(x)}\left(s_{1: n}\right) .
$$

Note that, indeed, the spatial operator $\frac{\partial^{2}}{\partial x^{2}}$ can be applied on $h_{n}^{(x)}\left(\right.$ and $H_{n}^{(x)}$ ) since it commutes with time operators (and their Laplace transform) ${ }^{1}$ :

$\overline{1}$ This holds under the standard hypotheses of the Lebesgue's dominated conver- 


$$
\begin{aligned}
\frac{\partial^{2} u(x, t)}{\partial x^{2}} & =\frac{\partial^{2}}{\partial x^{2}} \sum_{n=1}^{\infty} \int_{[0, t]^{n}} h_{n}^{(x)}\left(\tau_{1: n}\right) f\left(t-\tau_{1}\right) \ldots f\left(t-\tau_{n}\right) \mathrm{d} \tau_{1: n} \\
& =\sum_{n=1}^{\infty} \int_{[0, t]^{n}} \frac{\partial^{2} h_{n}^{(x)}\left(\tau_{1: n}\right)}{\partial x^{2}} f\left(t-\tau_{1}\right) \ldots f\left(t-\tau_{n}\right) \mathrm{d} \tau_{1: n} .
\end{aligned}
$$

Block (Vb): Similarly, the cascade of $\left\{H_{n}^{(x)}\left(s_{1: n}\right\}\right.$ and the linear spatial operator $\frac{\partial}{\partial x}$ defines the kernels $\frac{\partial H_{n}^{(x)}\left(s_{1: n}\right)}{\partial x}$. Then, from Eq. (19), the cascade with the square yields $\sum_{p=1}^{n-1} \frac{\partial}{\partial x} H_{p}^{(x)}\left(s_{1: p}\right) \frac{\partial}{\partial x} H_{n-p}^{(x)}\left(s_{p+1: n}\right)$. Finally, the cascade with the spatial integral operator $-\epsilon \int_{\Omega} \cdot \mathrm{d} x$ defines the Volterra series $\left\{B_{n}^{(x)}\right\}$ (see Fig.7(b)) with, for all $n \in \mathbb{N}^{*}$,

$$
B_{n}^{(x)}\left(s_{1: n}\right)=-\epsilon \int_{0}^{1} \sum_{p=1}^{n-1} \frac{\partial}{\partial x} H_{p}^{(x)}\left(s_{1: p}\right) \frac{\partial}{\partial x} H_{n-p}^{(x)}\left(s_{p+1: n}\right) \mathrm{d} x
$$

Block (Vc): The cascade of $H_{n}^{(x)}$ with $\frac{\partial^{2}}{\partial x^{2}}$ leads to the kernels

$$
C_{n}^{(x)}\left(s_{1: n}\right)=\frac{\partial^{2}}{\partial x^{2}} H_{n}^{(x)}\left(s_{1: n}\right)
$$

Block (Vd): For this case, $w_{d}(x, t)=-\phi(x) f(t)=\int_{-\infty}^{\infty} d_{1}^{(x)}\left(\tau_{1}\right) f\left(t-\tau_{1}\right) \mathrm{d} \tau_{1}$ with $d_{1}^{(x)}\left(\tau_{1}\right)=-\phi(x) \delta\left(\tau_{1}\right)$ where $\delta$ denotes the Dirac distribution (with Laplace transform 1). Hence, the corresponding Volterra kernels in the Laplace domain are given by

$$
D_{n}^{(x)}\left(s_{1: n}\right)=-\delta_{1, n} \phi(x)
$$

where $\delta_{1, n}$ denotes the Kronecker symbol $\left(\delta_{i, j}\right.$ equals 1 if $i=j$ and equals 0 else).

Using Eqs. (18) and (19), the Volterra kernels of the whole system in Fig. 7(b) are given by, for all $n \in \mathbb{N}^{*}, A_{n}^{(x)}\left(s_{1: n}\right)+\sum_{k=1}^{n-1} B_{k}^{(x)}\left(s_{1: k}\right) C_{n-k}^{(x)}\left(s_{k+1: n}\right)+D_{n}^{(x)}\left(s_{1: n}\right)$. Now, this system maps $f$ to 0 so that it defines the zero system for which all the Volterra kernels are zero. This leads to the equation satisfied by $H_{n}^{(x)}\left(s_{1: n}\right)$ inside $\Omega$, for all $n \in \mathbb{N}^{*}: \forall\left(x, s_{1: n}\right) \in \Omega \times\left(\mathbb{C}_{0}^{+}\right)^{n}$,

$$
\left[\Gamma\left(\widehat{s_{1: n}}\right)\right]^{2} H_{n}^{(x)}\left(s_{1: n}\right)-\frac{\partial^{2} H_{n}^{(x)}\left(s_{1: n}\right)}{\partial x^{2}}=\frac{E_{n}^{(x)}\left(s_{1: n}\right)}{1+\beta \widehat{s_{1: n}}}
$$

with

gence theorem (see e.g. [28]) and the Leibniz integral rule (see e.g. [25, (3.3.7)]). 


$$
\begin{aligned}
E_{1}^{(x)}\left(s_{1}\right) & =\phi(x) \\
E_{n}^{(x)}\left(s_{1: n}\right) & =\epsilon \sum_{\substack{p, q, r \geq 1 \\
p+q+r=n}} \int_{0}^{1}\left[\frac{\partial H_{p}^{(x)}\left(s_{1: p}\right)}{\partial x} \frac{\partial H_{q}^{(x)}\left(s_{p+1: p+q}\right)}{\partial x}\right] \mathrm{d} x \\
& \cdot \frac{\partial^{2} H_{r}^{(x)}\left(s_{p+q+1: n}\right)}{\partial x^{2}} \text { if } n \geq 2 .
\end{aligned}
$$

and $\forall s \in \mathbb{C}_{0}^{+}, \quad \Gamma(s)=\sqrt{\frac{s^{2}+\alpha s}{1+\beta s}}$.

For each $n \in \mathbb{N}^{*}$, Eq. (22) is a linear second order differential equation on $H_{n}^{(x)}$ with respect to $x$. Indeed, kernels $E_{n}^{(x)}$ only involve $H_{p, q, r}^{(x)}$ with $p, q, r \leq n-1$.

\subsubsection{Equations satisfied by the kernels at the boundaries.}

The boundary conditions Eq. (9) impose a null displacement at $x \in\{0 ; 1\}$. This means that kernels $h_{n}^{(x)}$ are zero at $x \in\{0 ; 1\}$ (see Fig. 8). In the Laplace

$$
f(t) \quad-\left\{h_{n}^{(x=0)}\right\} u(x=0, t)=0
$$

Fig. 8. Boundary condition on $h_{n}^{(x)}$ at $x=0$ (the same condition holds at $x=1$ ). domain, this yields, for all $n \in \mathbb{N}^{*}$ :

$$
\forall\left(x, s_{1: n}\right) \in\{0 ; 1\} \times\left(\mathbb{C}_{0}^{+}\right)^{n}, \quad H_{n}^{(x)}\left(s_{1: n}\right)=0 .
$$

\subsection{Solving the Volterra kernels}

\subsubsection{Analytic solution}

For each $n \in \mathbb{N}^{*}$, Eqs. (22) to (26) define a linear boundary value problem, the solution of which is given by (see apdx. 9.1),

$$
\forall\left(x, s_{1: n}\right) \in \Omega \times\left(\mathbb{C}_{0}^{+}\right)^{n}, \quad H_{n}^{(x)}\left(s_{1: n}\right)=\int_{\Omega} G\left(x, \xi, \widehat{s_{1: n}}\right) E_{n}^{(\xi)}\left(s_{1: n}\right) \mathrm{d} \xi
$$

where, for all $(x, \xi, s) \in \Omega \times \Omega \times \mathbb{C}_{0}^{+}$,

$$
G(x, \xi, s)=\frac{\cosh ((1+x+\xi) \Gamma(s))-\cosh ((1-|x-\xi|) \Gamma(s))}{2(1+\beta s) \Gamma(s) \sinh \Gamma(s)},
$$

Note that $G$ does not depend on the choice of the square root for $\Gamma$ since $\Gamma \mapsto-\Gamma$ keeps $G$ invariant. 
For $n=1$, this solution is exactly that of the linearized problem ((M1) with $\epsilon=0)$ where the Green function $G$ is applied on the spatial distribution $E_{1}^{(x)}\left(s_{1}\right)=\phi(x)$ in the Laplace domain. For $n \geq 2, E_{n}^{(x)}$ involves kernels $H_{p}^{(x)}$ with $p \leq n-1$ (see Eq. (24)) so that Eq. (27) is a recurrence equation which makes the explicit derivation of analytic expressions possible. Nevertheless, in the following, we choose to use a modal decomposition: it helps to simplify these recurrent integral equations into recurrent algebraic equations.

\subsubsection{Modal decomposition}

For each $n \in \mathbb{N}^{*}$, the linear boundary value problem Eqs. (22) to (26) admits an orthonormal basis of eigen-functions $\mathcal{B}=\left\{e_{k}\right\}_{k \in \mathbb{N}^{*}}$ on the Hilbert space $L^{2}(\Omega)$ (see e.g. [29]). Functions $e_{k}$ which define the spatial modes are

$$
\forall(k, x) \in \mathbb{N}^{*} \times \Omega, \quad e_{k}(x)=\sqrt{2} \sin (k \pi x) .
$$

They satisfy: (i) the Dirichlet boundary conditions; (ii) $\frac{\partial^{2} e_{k}}{\partial x^{2}}=-(k \pi)^{2} e_{k}$; (iii) for all $(i, j) \in\left(\mathbb{N}^{*}\right)^{2}\left\langle e_{i}, e_{j}\right\rangle=\delta_{i, j}$ (Kronecker symbol) where the scalar product on $L^{2}(\Omega)$ is defined by $\forall(f, g) \in\left(L^{2}(\Omega)\right)^{2},\langle f, g\rangle=\int_{\Omega} f(x) g(x) \mathrm{d} x$.

Consider the decomposition of $H_{n}^{(x)}$ on $\mathcal{B}$, given by, for all $n \in \mathbb{N}^{*}$,

$$
\forall\left(s_{1: n}\right) \in\left(\mathbb{C}_{0}^{+}\right)^{n}, \quad H_{n}^{(x)}\left(s_{1: n}\right) \underset{\overline{L^{2}}}{=} \sum_{k \in \mathbb{N}^{*}} H_{n}^{[k]}\left(s_{1: n}\right) e_{k}(x),
$$

where $H_{n}^{[k]}=\left\langle H_{n}^{(x)}, e_{k}\right\rangle$ denotes the projection of $H_{n}^{(x)}$ on $e_{k}$. The relations satisfied by $H_{n}^{[k]}$ are obtained by projecting Eqs. (22) to (24) on $\mathcal{B}$, as detailed in apdx. 9.2. This yields the following algebraic equations, for all $(n, k) \in\left(\mathbb{N}^{*}\right)^{2}$, $\left(s_{1: n}\right) \in\left(\mathbb{C}_{0}^{+}\right)^{n}$,

$$
\begin{aligned}
H_{n}^{[k]}\left(s_{1: n}\right) & =Q^{[k]}\left(\widehat{s_{1: n}}\right) E_{n}^{[k]}\left(s_{1: n}\right), \\
E_{1}^{[k]}\left(s_{1}\right) & =\left\langle\phi, e_{k}\right\rangle=\phi_{k}, \\
E_{n}^{[k]}\left(s_{1: n}\right) & =-\epsilon k^{2} \pi^{4} \sum_{\substack{p, q, r \geq 1 \\
p+q+r=n}}\left[\sum_{\ell \in \mathbb{N}^{*}} \ell^{2} H_{p}^{[\ell]}\left(s_{1: p}\right) H_{q}^{[\ell]}\left(s_{p+1: p+q}\right)\right] H_{r}^{[k]}\left(s_{p+q+1: n}\right), \text { if } n \geq 2 .
\end{aligned}
$$

where $Q^{[k]}(s)$ is the rational function given by

$$
Q^{[k]}(s)=\left[s^{2}+\left(\alpha+\beta k^{2} \pi^{2}\right) s+k^{2} \pi^{2}\right]^{-1},
$$

which is analytic in $\mathbb{C}_{0}^{+}$. Indeed, for $\alpha \geq 0$ and $\beta \geq 0$, a straightforward analysis proves the real parts of the poles of $Q^{[k]}$ are all negative (or zero for the particular case $\alpha=\beta=0$ ). More precisely, these poles correspond to damped oscillating modes if $1 \leq k<K^{\star}=22690$ (complex conjugated pair of 
poles) and purely evanescent ones if $k \geq K^{\star}$ (real negative poles). A detailed study of such an analysis can be found in [30] for the case of a bar with similar dampings. Fig. 9 represents the poles in the Laplace complex plane, for the typical values $\alpha$ and $\beta$ given in Table 1 .

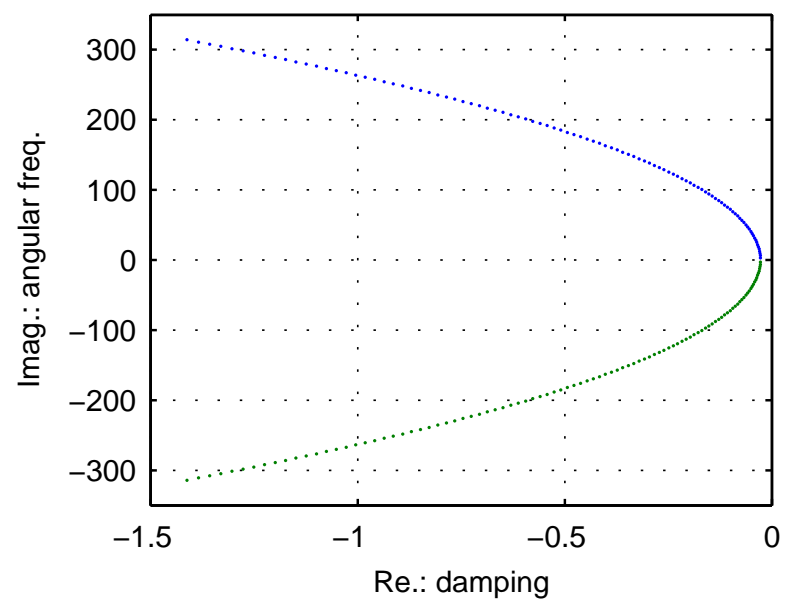

Fig. 9. Poles of $Q^{[k]}$ for $1 \leq k \leq K=100$ and $(\alpha, \beta)$ given in Table 1 .

Computing the analytic expressions for orders $n \in[1,5]_{\mathbb{N}}$ leads to, $\forall\left(k, s_{1: n}\right) \in$ $\mathbb{N}^{*} \times\left(\mathbb{C}_{0}^{+}\right)^{n}$

$$
\begin{aligned}
& H_{1}^{[k]}\left(s_{1}\right)=\phi_{k} Q^{[k]}\left(s_{1}\right), \\
& H_{2}^{[k]}\left(s_{1: 2}\right)=0, \\
& H_{3}^{[k]}\left(s_{1: 3}\right)=-\epsilon k^{2} \pi^{4} Q^{[k]}\left(\widehat{s_{1: 3}}\right) {\left[\sum_{\ell \in \mathbb{N}^{*}} \ell^{2} H_{1}^{(\ell)}\left(s_{1}\right) H_{1}^{(\ell)}\left(s_{2}\right)\right] H_{1}^{(k)}\left(s_{3}\right) . } \\
& H_{4}^{[k]}\left(s_{1: 4}\right)=0, \\
& H_{5}^{[k]}\left(s_{1: 5}\right)=-\epsilon k^{2} \pi^{4} Q^{[k]}\left(\widehat{s_{1: 5}}\right)\left[\sum_{\ell \in \mathbb{N}^{*}} \ell^{2} H_{3}^{[\ell]}\left(s_{1: 3}\right) H_{1}^{[\ell]}\left(s_{4}\right) H_{1}^{[k]}\left(s_{5}\right)\right. \\
&+\sum_{\ell \in \mathbb{N}^{*}} \ell^{2} H_{1}^{[\ell]}\left(s_{1}\right) H_{3}^{[\ell]}\left(s_{2: 4}\right) H_{1}^{[k]}\left(s_{5}\right) \\
&\left.+\sum_{\ell \in \mathbb{N}^{*}} \ell^{2} H_{1}^{[\ell]}\left(s_{1}\right) H_{1}^{[\ell]}\left(s_{2}\right) H_{3}^{[k]}\left(s_{3: 5}\right)\right]
\end{aligned}
$$

Remark 5: For $n=2$, the sum on $p, q, r \geq 1$ s.t. $p+q+r=n$ is empty so that, from Eq. (33), $E_{n}^{[k]}$ and $H_{n}^{(x)}$ are zero. More generally, a recurrence proves that for all $m \in \mathbb{N}^{*}, H_{2 m}^{(x)}=0$ and $H_{2 m+1}^{(x)}$ is proportional to $\epsilon^{m}$.

Remark 6: For $n=1$, the solution leads to the standard modal decomposition of the linearized problem. For orders $n \geq 2$, Eqs. (31), (33) and (34) show how the dynamics of lower orders $(1 \leq p, q, r \leq n-1$ s.t. $p+q+r=n)$ generate the nonlinear dynamics of order $n$. Eq. (33) also indicates what spatial modes 
contribute to the dynamics of order $n$ of the mode $k$ : all the modes $\ell \in \mathbb{N}^{*}$ contribute to the elongation (through the orders $p$ and $q$ ) while the dynamics of order $r$ (which contribute to the laplacian) are only those of the mode $k$.

\subsubsection{Combinatorics, trees, and physical interpretations}

The combinatorics due to Eqs. (30) to (33) can be reorganized as one summation of elementary terms, the indexes of summation being naturally described by full ${ }^{(*)}$ ternary $^{(* *)}$ trees $^{2}$, as detailed below.

\section{Definition 1}

Let $\mathbb{A}_{n}$ be the sets of ternary trees defined by, for $n \in \mathbb{N}^{*}$,

$$
\begin{array}{cl}
\mathbb{A}_{n}=\mathbb{N}^{*}, & \text { if } n=1, \\
\mathbb{A}_{n}=\emptyset, & \text { if } n \text { is even, } \\
\mathbb{A}_{n}=\bigcup_{\substack{p, q, r \geq 1 \\
p, q, r \text { odd } \\
p+q+r=n}}\left\{\left(\mathfrak{a}_{1}, \mathfrak{a}_{2}, \mathfrak{a}_{3}\right) \in \mathbb{A}_{p} \times \mathbb{A}_{q} \times \mathbb{A}_{r} \mid \mathfrak{k}\left(\mathfrak{a}_{1}\right)=\mathfrak{k}\left(\mathfrak{a}_{2}\right)\right\}, & \text { if } n \geq 3 \text { is odd } \\
&
\end{array}
$$

where $\mathfrak{k}(\mathfrak{a})$ is the right-sided leaf of $\mathfrak{a}$ : for $\mathfrak{a}=k \in \mathbb{A}_{1}, \mathfrak{k}(\mathfrak{a})=k$; for $\mathfrak{a}=$ $\left(\mathfrak{a}_{1}, \mathfrak{a}_{2}, \mathfrak{a}_{3}\right) \in \mathbb{A}_{n}$ (with $n \geq 3$ ), $\mathfrak{k}(\mathfrak{a})=\mathfrak{k}\left(\mathfrak{a}_{3}\right)$. Moreover, $\mathfrak{n}(\mathfrak{a})$ denotes the number of leaves of $\mathfrak{a}$ so that if $\mathfrak{a} \in \mathbb{A}_{n}$ and if $n$ is odd, $\mathfrak{n}(\mathfrak{a})=n$.

Examples: $\begin{aligned} & \mathfrak{a} \\ & 1 \\ & 8\end{aligned}$ denotes the tree $\mathfrak{a} \in \mathbb{A}_{1}$ such that $\mathfrak{k}(\mathfrak{a})=8$.

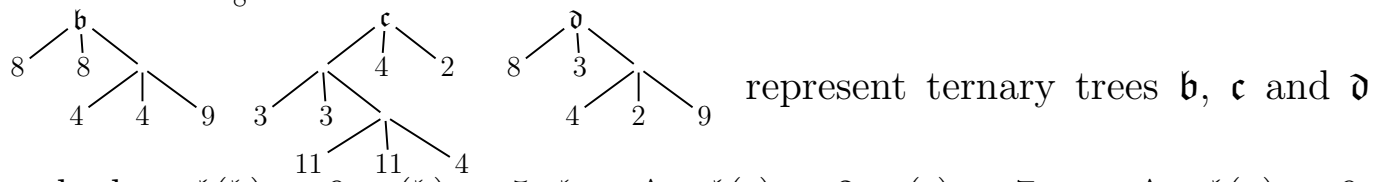

such that: $\mathfrak{k}(\mathfrak{b})=9, \mathfrak{n}(\mathfrak{b})=5, \mathfrak{b} \in \mathbb{A}_{5} ; \mathfrak{k}(\mathfrak{c})=2, \mathfrak{n}(\mathfrak{c})=7, \mathfrak{c} \in \mathbb{A}_{7} ; \mathfrak{k}(\mathfrak{d})=9$, $\mathfrak{n}(\mathfrak{d})=5$ but note that $\mathfrak{d} \notin \mathbb{A}_{5}$. Indeed, $\mathfrak{d}=\left(\mathfrak{d}_{1}, \mathfrak{d}_{2}, \mathfrak{d}_{3}\right)$ with $\mathfrak{d}_{1}=8, \mathfrak{d}_{2}=3$ and $\mathfrak{d}_{3}=(4,2,9)$ so that, in Eq. (42), the condition $\mathfrak{k}\left(\mathfrak{a}_{1}\right)=\mathfrak{k}\left(\mathfrak{a}_{2}\right)$ is not satisfied (twice): first, because $\mathfrak{k}\left(\mathfrak{d}_{1}\right)=4 \neq 3=\mathfrak{k}\left(\mathfrak{d}_{2}\right)$; second, because $\mathfrak{d}_{3} \notin \mathbb{A}_{3}$ for a similar reason $(4 \neq 2)$.

\section{Theorem 1}

For all $n \in \mathbb{N}^{*}$, kernels $H_{n}^{(x)}$ are given by, for all $\left(s_{1: n}\right) \in\left(\mathbb{C}_{0}^{+}\right)^{n}$,

$$
H_{n}^{(x)}\left(s_{1: n}\right)=\sum_{\mathfrak{a} \in \mathbb{A}_{n}} H_{\mathfrak{a}}\left(s_{1: n}\right) e_{\mathfrak{k}(\mathfrak{a})}(x)
$$

where, for all $\mathfrak{a} \in \mathbb{A}_{n}$ (with $n$ odd),

$\overline{2}$ This is linked to the nonlinearity of (M1) which is homogeneous ${ }^{(*)}$ of degree $3^{(* *)}$. Recall that a ternary tree is a tree in which every node has at most three children. A full ternary tree is a tree in which every node has zero or three children. 


$$
\begin{gathered}
\text { if } n=1, \quad H_{\mathfrak{a}}\left(s_{1}\right)=\phi_{\mathfrak{k}(\mathfrak{a})} Q^{[\mathfrak{k}(\mathfrak{a})]}\left(s_{1}\right), \\
\text { if } n \geq 3, \quad H_{\mathfrak{a}}\left(s_{1: n}\right)=-\epsilon\left[\mathfrak{k}\left(\mathfrak{a}_{1}\right) \mathfrak{k}\left(\mathfrak{a}_{3}\right) \pi^{2}\right]^{2} Q^{[\mathfrak{k}(\mathfrak{a})]}\left(\widehat{s_{1: n}}\right) H_{\mathfrak{a}_{1}}\left(s_{1: \mathfrak{n}\left(\mathfrak{a}_{1}\right)}\right) \\
\cdot H_{\mathfrak{a}_{2}}\left(s_{\mathfrak{n}\left(\mathfrak{a}_{1}\right)+1: \mathfrak{n}\left(\mathfrak{a}_{1}\right)+\mathfrak{n}\left(\mathfrak{a}_{2}\right)}\right) H_{\mathfrak{a}_{3}}\left(s_{\mathfrak{n}\left(\mathfrak{a}_{1}\right)+\mathfrak{n}\left(\mathfrak{a}_{2}\right)+1: n}\right) . \\
\text { with } \mathfrak{a}=\left(\mathfrak{a}_{1}, \mathfrak{a}_{2}, \mathfrak{a}_{3}\right),
\end{gathered}
$$

The proof of this theorem is detailed in apdx. 9.3.

Interpretation: The trees memorize and isolate each elementary inheritance that each modal linear kernels transmit to a $n$-th order kernel. For example, kernel $H_{\mathfrak{e}}$ with $\mathfrak{e}=(4,4,9) \in \mathbb{A}_{n}$ and $n=3$ models how the elongation due to the linear dynamics of the mode 4 acts on the linear dynamics of mode 9 and contributes to its nonlinear dynamics of order $n=3$. Kernel $H_{\mathfrak{b}}\left(\mathfrak{b}=(8,8, \mathfrak{e}) \in \mathbb{A}_{n}\right.$ with $\left.n=5\right)$ models how that of mode 8 acts on the dynamics due to $H_{\mathfrak{e}}$ (order 3 , mode 9 ) and contributes to the dynamics (mode 9) of order $n=5$. More generally, for $\mathfrak{f}=\left(\mathfrak{f}_{1}, \mathfrak{f}_{2}, \mathfrak{f}_{3}\right) \in \mathbb{A}_{n}(n \geq 3$, odd $)$, $H_{\mathfrak{f}}$ models how the elongation introduced by the couple of dynamics due to $\left(H_{\mathfrak{f}_{1}}, H_{\mathfrak{f}_{1}}\right)$ for the same the mode $k=\mathfrak{k}\left(\mathfrak{f}_{1}\right)=\mathfrak{k}\left(\mathfrak{f}_{2}\right)$ acts on the dynamics due to $H_{\mathfrak{f}_{3}}$ (order $\mathfrak{n}\left(\mathfrak{f}_{3}\right)$, mode $\left.\mathfrak{k}\left(\mathfrak{f}_{3}\right)\right)$ and contributes to the dynamics of order $n$ of the mode $\mathfrak{k}(\mathfrak{f})=\mathfrak{k}\left(\mathfrak{f}_{3}\right)$.

Remark 7: Note that the condition $\mathfrak{k}\left(\mathfrak{a}_{1}\right)=\mathfrak{k}\left(\mathfrak{a}_{2}\right)$ in the definition Eq. (42) is due to the integral in Eq. (24) and the orthogonality of $\mathcal{B}=\left\{e_{k}\right\}_{k \in \mathbb{N}^{*}}$.

\subsection{Identification of realizable structures for the sound synthesis}

Computing the string dynamics from Eq. (21) using time-domain versions of Eqs. (43) to (45) leads to an infinite algorithmic complexity. In practice, infinite sums are truncated. First, only a finite number of modes are considered using $\widetilde{\mathbb{A}}_{1}=[1, K]_{\mathbb{N}}$ in place of $\mathbb{A}_{1}$ in definition 1 . This yields good approximations in Eq. (43), if modes higher than $K$ can be dropped due to negligible $\phi_{k}=\left\langle\phi, e_{k}\right\rangle$, or even, is exact if $\phi_{k}=0$ for $k>K$. Second, only the first kernels $H_{n}^{(x)}$ for $1 \leq n \leq N=2 M+1$ are kept in Eq. (21). This defines the approximation $\breve{u}_{N}(x, t)$ such that $u(x, t)=\breve{u}_{N}(x, t)+o\left(\epsilon^{M}\right)$ (see remark 5).

The realization theory allows to build structures which are well-adapted to the numerical simulation. For Volterra kernels, realizations can be performed from the regular Volterra kernels (see [23, chap.4] for a detailed presentation). Here, an alternative identification is proposed in theorem 2, which does not require to compute the regular kernels. Then, realizations are deduced and detailed for orders $n=1,3,5$. 


\section{Definition 2 (Homogeneous Volterra system)}

A Volterra system $\mathcal{S}$ with kernels $\left\{h_{n^{\prime}}\right\}_{n^{\prime} \in \mathbb{N}^{*}}$ is said to be "homogeneous" of order $n \in \mathbb{N}^{*}$, if $h_{n}$ is non-zero, and if, for all $n^{\prime} \in \mathbb{N}^{*} \backslash\{n\}$, the kernels $h_{n^{\prime}}$ are null.

Note that linear filters are homogeneous systems of order 1.

\section{Definition 3 (Elementary ternary Volterra system)}

A system $\mathcal{S}$ with input $f$ and output $u$ is an "elementary ternary Volterra system" if

- it exists three homogeneous Volterra systems $\mathcal{S}_{a}, \mathcal{S}_{b}, \mathcal{S}_{c}$, of respective order $p, q, r$, with respective output $y_{a}, y_{b}, y_{c}$, and all fed by the same input $f$,

- it exists a linear system $\mathcal{S}_{d}$,

such that $u$ is the output of $\mathcal{S}_{d}$ fed by the input $w(t)=y_{a}(t) y_{b}(t) y_{c}(t)$ (see Fig. 10).

From interconnection laws Eqs.(19) and (20), the system $\mathcal{S}$ is proven to be homogeneous of order $n=p+q+r$ : its kernel of order $n$ is given by

$$
H_{n}\left(s_{1: n}\right)=A_{p}\left(s_{1: p}\right) B_{q}\left(s_{p+1: p+q}\right) C_{r}\left(s_{p+q+1: n}\right) D_{1}\left(\widehat{s_{1: n}}\right),
$$

in the Laplace domain, where $a_{p}, b_{q}, c_{r}$, and $d_{1}$ denote the non-zero kernels of systems $\mathcal{S}_{a}, \mathcal{S}_{b}, \mathcal{S}_{c}$, and $\mathcal{S}_{d}$, respectively.

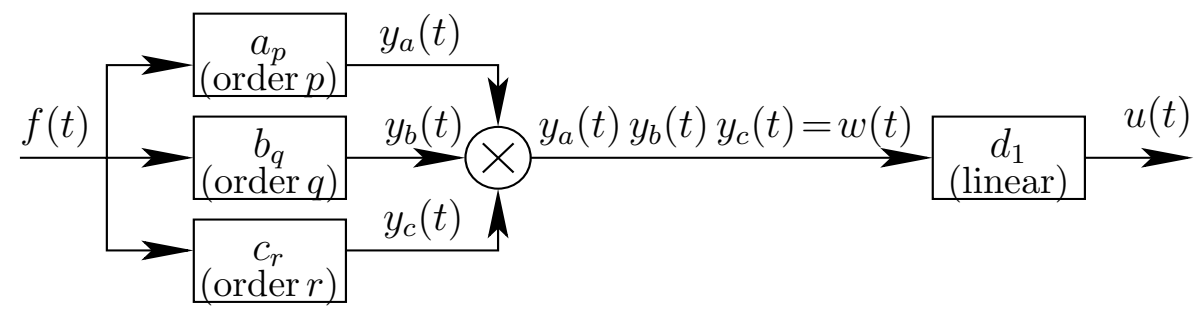

Fig. 10. Elementary ternary Volterra system of homogeneous order $n=p+q+r$.

\section{Theorem 2 (Structure composed of filters, sums, and products)}

The solution $u(x, t)$ of (M1) obtained from Eq. (21) and (43) to (45) is given by the sum

$$
u(x, t)=\sum_{n \in \mathbb{N}^{*}} \sum_{\mathfrak{a} \in \mathbb{A}_{n}} u_{\mathfrak{a}}(t) e_{\mathfrak{k}(\mathfrak{a})}(x)
$$

where the sub-dynamics signals $u_{\mathfrak{a}}$ can be realized using linear filters and products, as follows:

- if $\mathfrak{a} \in \mathbb{A}_{1}$, then $u_{\mathfrak{a}}$ is the output of the linear filter with transfer function $H_{\mathfrak{a}}(s)$ given by Eq. (44); 
- if $n \geq 3$ is odd and if $\mathfrak{a}=\left(\mathfrak{a}_{1}, \mathfrak{a}_{2}, \mathfrak{a}_{3}\right) \in \mathbb{A}_{n}$, then $u_{\mathfrak{a}}$ is the output of the linear filter with transfer function

$$
G_{\mathfrak{a}}(s)=-\epsilon\left[\mathfrak{k}\left(\mathfrak{a}_{1}\right) \mathfrak{k}\left(\mathfrak{a}_{3}\right) \pi^{2}\right]^{2} Q^{[\mathfrak{k}(\mathfrak{a})]}(s)
$$

fed by the input $v_{\mathfrak{a}}(t)=u_{\mathfrak{a}_{1}}(t) u_{\mathfrak{a}_{2}}(t) u_{\mathfrak{a}_{3}}(t)$ (see Fig. 11)

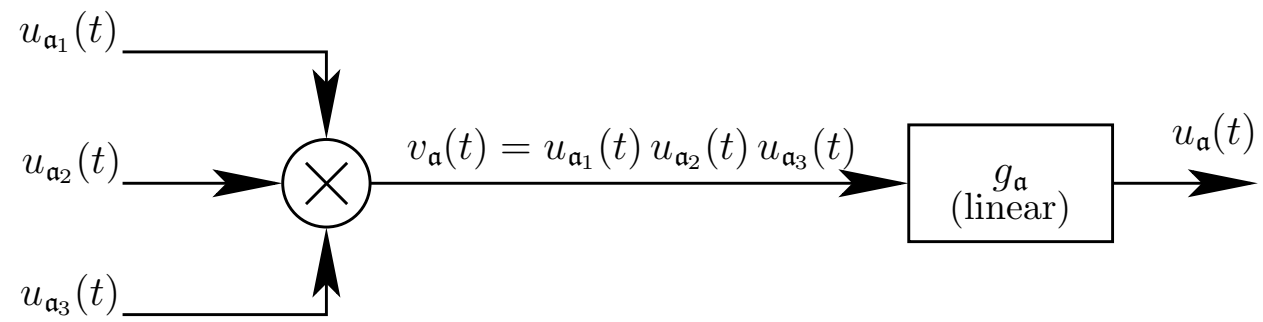

Fig. 11. Realization of $u_{\mathfrak{a}}$ for $\mathfrak{a}=\left(\mathfrak{a}_{1}, \mathfrak{a}_{2}, \mathfrak{a}_{3}\right) \in \mathbb{A}_{n}$ with odd $n \geq 3: g_{\mathfrak{a}}$ is the convolution kernel of the filter associated to the transfer function $G_{\mathfrak{a}}$.

\section{Proof}

The identification is straightforward if $n=1$ or if $n$ is even. Now, let $n \geq 3$ be odd. Let $\mathfrak{a}=\left(\mathfrak{a}_{1}, \mathfrak{a}_{2}, \mathfrak{a}_{3}\right) \in \mathbb{A}_{n}$. Then, $H_{\mathfrak{a}}$ (see Eq. (45)) has the form Eq. (46) with $p=\mathfrak{n}\left(\mathfrak{a}_{1}\right), q=\mathfrak{n}\left(\mathfrak{a}_{2}\right), r=\mathfrak{n}\left(\mathfrak{a}_{3}\right)$, and $A_{p}=H_{\mathfrak{a}_{1}}, B_{q}=H_{\mathfrak{a}_{2}}, C_{r}=H_{\mathfrak{a}_{3}}$, $D_{1}=G_{\mathfrak{a}}$ (see Eq. (48)). The identification of the realizable structure arises from the definition 3.

\subsubsection{Application: building realizable structures for orders $n=1,3,5$}

In practice, the sound synthesis is performed for a finite number of modes $k \in \widetilde{\mathbb{A}}_{1}=[1, K]_{\mathbb{N}}$ and a finite number $N$ of kernels. The solution $u(x, t)$ given by Eq. (47) is approximated by

$$
\begin{aligned}
\breve{u}_{N}(x, t) & =\sum_{n=1}^{N} \sum_{\mathfrak{a} \in \widetilde{\mathbb{A}}_{n}} u_{\mathfrak{a}}(t) e_{\mathfrak{k}(\mathfrak{a})}(x)=\sum_{n=1}^{N} \sum_{k=1}^{K} u_{n}^{[k]}(t) e_{k}(x), \\
\text { with } u_{n}^{[k]}(t) & =\sum_{\mathfrak{a} \in \widetilde{\mathbb{A}}_{n}(k)} u_{\mathfrak{a}}(t), \\
\text { and } \widetilde{\mathbb{A}}_{n}(k) & =\left\{\mathfrak{a} \in \widetilde{\mathbb{A}}_{n} \mid \mathfrak{k}(\mathfrak{a})=k\right\}
\end{aligned}
$$

In this section, realizations of $u_{n}^{[k]}$ and then of $\breve{u}_{N}(x, t)$ are derived using the theorem 2 .

For $n=1$ and $\mathfrak{a}=k \in \widetilde{\mathbb{A}}_{1}=[1, K]_{\mathbb{N}}, u_{\mathfrak{a}}=u_{1}^{[k]}$ is the output of the linear filter with kernel $h_{1}^{[k]}=\phi_{k} q^{[k]}$ fed by $f(t)$ (see theorem 2 and Eq.(44)). The static gain $\phi_{k}$ (see Fig.12@) measures how much the mode $e_{k}$ is excited by the spatial distribution $\phi$. 
For $n=3, \mathfrak{a} \in \widetilde{\mathbb{A}}_{3} \subset\left(\widetilde{\mathbb{A}}_{1}\right)^{3}$ has the form $\mathfrak{a}=(\ell, \ell, k)$. From theorem 2 and Fig. 11, $u_{\mathfrak{a}}(t)$ is the output of the linear filter with kernel $g_{\mathfrak{a}}=-\ell^{2} \gamma_{k} q^{[k]}$ fed by $u_{1}^{[\ell]}(t) u_{1}^{[\ell]}(t) u_{1}^{[k]}(t)$, where $\gamma_{k}$ denotes

$$
\gamma_{k}=\epsilon k^{2} \pi^{4}
$$

Separating the factors depending on $\ell$ from those depending on $k$ leads to the realization in Fig. 12(b)). The realization of $u_{3}^{[k]}\left(k \in[1, K]_{\mathbb{N}}\right)$ is obtained by

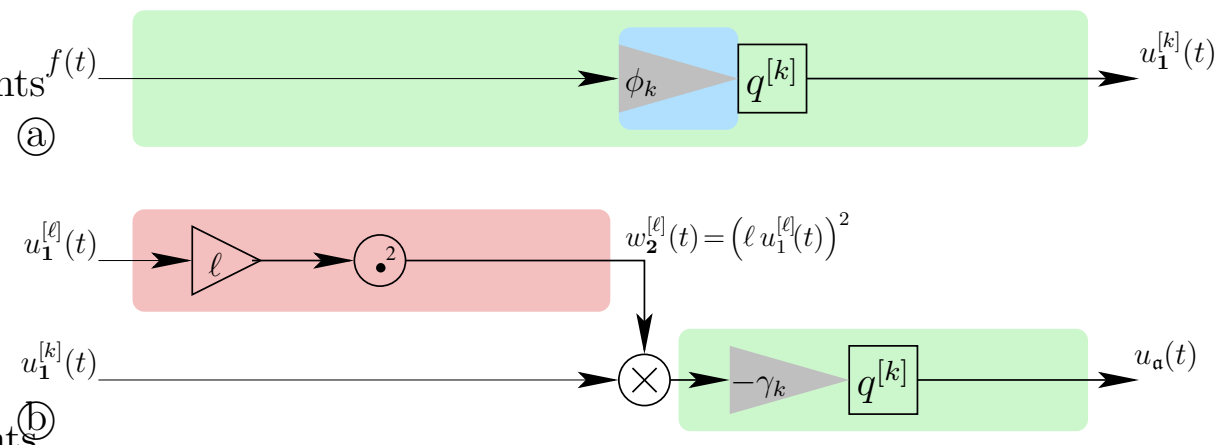

Fig. 12. (a): Realization of $u_{1}^{[k]}$; (b): Realization of $u_{\mathfrak{a}}$ with $\mathfrak{a}=(\ell, \ell, k) \in \widetilde{\mathbb{A}}_{3}$.

summing the outputs $u_{\mathfrak{a}}(t)$ of realizations of Fig. 12(b) over $\widetilde{\mathbb{A}}_{3}(k)=\{(\ell, \ell, k) \mid \ell \in$ $\left.\widetilde{\mathbb{A}}_{1}\right\}$, following Eqs. (50) and (51). Collecting all these contributions over $\ell$ and factorizing by the common linear filter $-\gamma_{k} q^{[k]}$ yield the concise realization given in Fig. 13. As a consequence, the approximated solution $\breve{u}_{3}(x, t)$

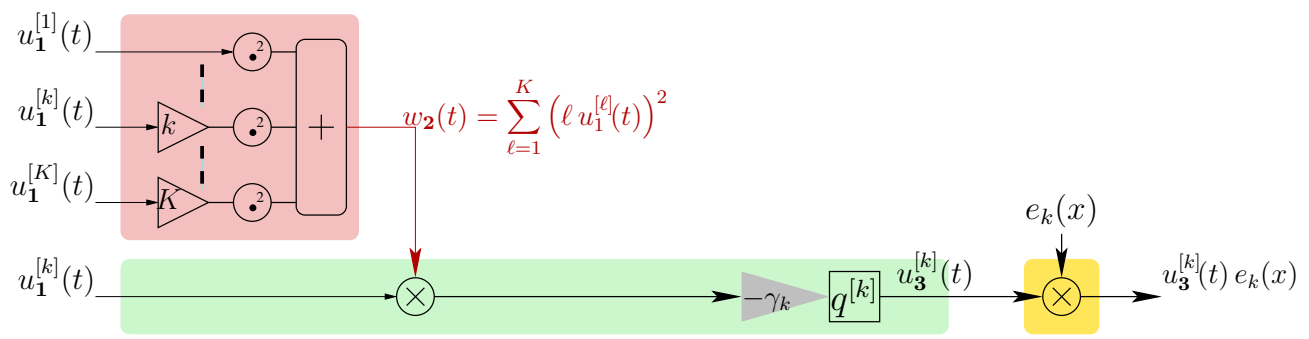

Fig. 13. Realization of $u_{3}^{[k]}$ with its associated modal shape $u_{3}^{[k]}(t) e_{k}(x)$.

in Eq. (49) can be realized as detailed in Fig.14. In this structure, kernels $q^{[k]}$ are the impulse responses of second order AR-filters associated to the transfer functions $Q^{[k]}(s)$. If $N_{q}^{+}$denotes the number of sums involved in a digital simulation of $q^{[k]}$ (similarly, $N_{q}^{\times}$for products), the global complexity can be evaluated, as detailed in Table 2. Digital implementations of linear filters involved in Fig. 14 are very standard (see e.g. [32]). Here, a method which preserves the exact eigen-frequencies and the exact dampings is proposed (see details in apdx.9.4). It leads to the parameters $N_{q}^{+}=4, N_{q}^{\times}=5$.

Nevertheless, the products (in the time domain) of $N=3$ signals with a frequency range $\left[0, f^{*}\right]$ yield a signal with frequency range $\left[0, N f^{\star}\right]$. Theoretically, rejecting the aliasing due to sampling with frequency $f_{s}$ requires that 


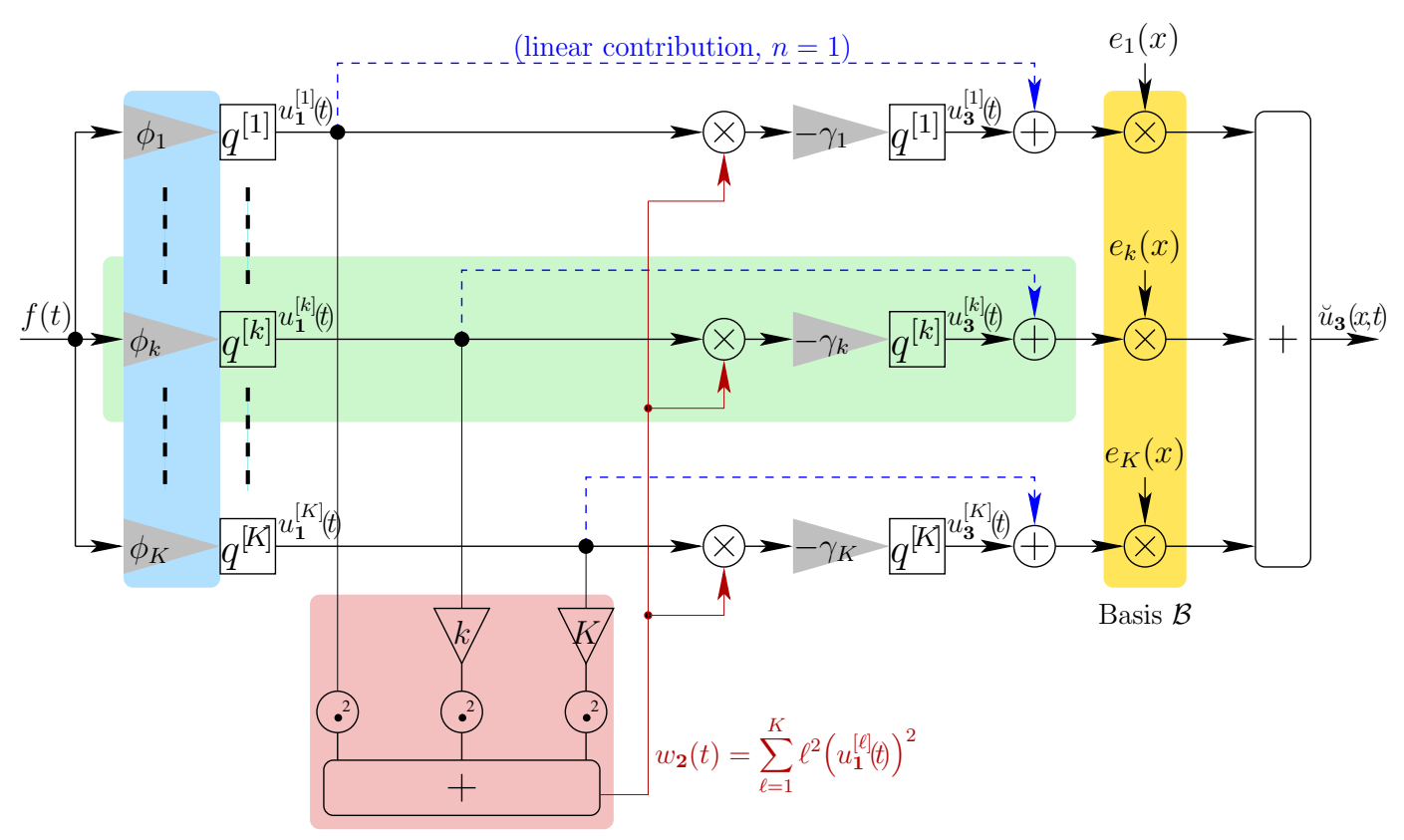

Fig. 14. Block-diagram of an $o(\epsilon)$-simulation of (M1) with $K$ modes: the dashed arrows isolate the linear dynamics $(n=1)$ of each mode; the shaded central part isolates the $o(\epsilon)$-dynamics of a mode $k$; the shaded bottom part isolates the dynamics of the integral term in (M1) (gains $1, k$ and $K$ before the square operators are due to $\left.\frac{\partial}{\partial x}\right)$; the shaded left part corresponds to simple gains, controlled by the spatial distribution of the excitation force.

\begin{tabular}{|l||l|l|}
\cline { 2 - 3 } \multicolumn{1}{c||}{} & linear approx.: $N=1, o\left(\epsilon^{0}\right)$ & third order approx.: $N=3, o\left(\epsilon^{1}\right)$ \\
\hline \hline$N^{+}$ & $K\left(N_{q}^{+}+N_{x}\right)-N_{x}$ & $K\left(2 N_{q}^{+}+N_{x}+2\right)-N_{x}-1$ \\
\hline$N^{\times}$ & $K\left(N_{q}^{\times}+N_{x}\right)$ & $K\left(2 N_{q}^{\times}+N_{x}+3\right)-1$ \\
\hline$N^{\text {flops }}$ & $K\left(N_{q}^{\text {flops }}+2 N_{x}\right)-N_{x}$ & $K\left(2 N_{q}^{\text {flops }}+2 N_{x}+5\right)-N_{x}$ \\
\hline
\end{tabular}

Table 2

Number of floating point operations to compute $u(x, t)$ for (M1) with $K$ modes, at a given time, and at $N_{x}$ observation points (see $\S 7$ for typical values): the number of sums $\left(N^{+}\right)$, products $\left(N^{\times}\right)$, and flops $\left(N^{\text {flops }}=N^{+}+N^{\times}\right)$are given for the linear and the third order approximations.

$f_{s} / 2>N f^{\star}$ (Shannon-Nyquist theorem). Here, the second order filters with impulse response $q^{[k]}$ significantly cut off the spectrum beyond $f_{k}\left[1+1 / Q_{k}\right]$ where the quality factor is $Q_{k}=\frac{k \pi}{\alpha+\beta k^{2} \pi^{2}}$. As a consequence,

$$
\frac{f_{s}}{2}>N f_{K}\left(1+\frac{1}{Q_{K}}\right)
$$

is sufficient in practice. This condition will be used for the sound synthesis in $\S 7$.

For $n=5$, the identification given by theorem 2 yields the realization given in 
Fig. 15. Note that this structure can be also identified using Eq. (39): the first factor in Eq. (39) accounts for the filter with kernel $-\gamma_{k} q^{[k]}$ which is factorized in the realization (similarly to Fig. 13 for the case $n=3$; in the second factor, the two first terms describes twice the same sub-system which corresponds to the part involving $w_{4}(t)$ and the gain 2 in Fig. 15; finally, the third term describes the sub-system corresponding to the bottom part involving $u_{3}^{[k]}(t)$ and $w_{2}(t)$. The realization given in Fig. 15 allows to complement Fig. 14, yielding a realization of $\breve{u}_{5}(x, t)$. Numerical simulations of $\breve{u}_{3}$ and $\breve{u}_{5}$ and comparisons

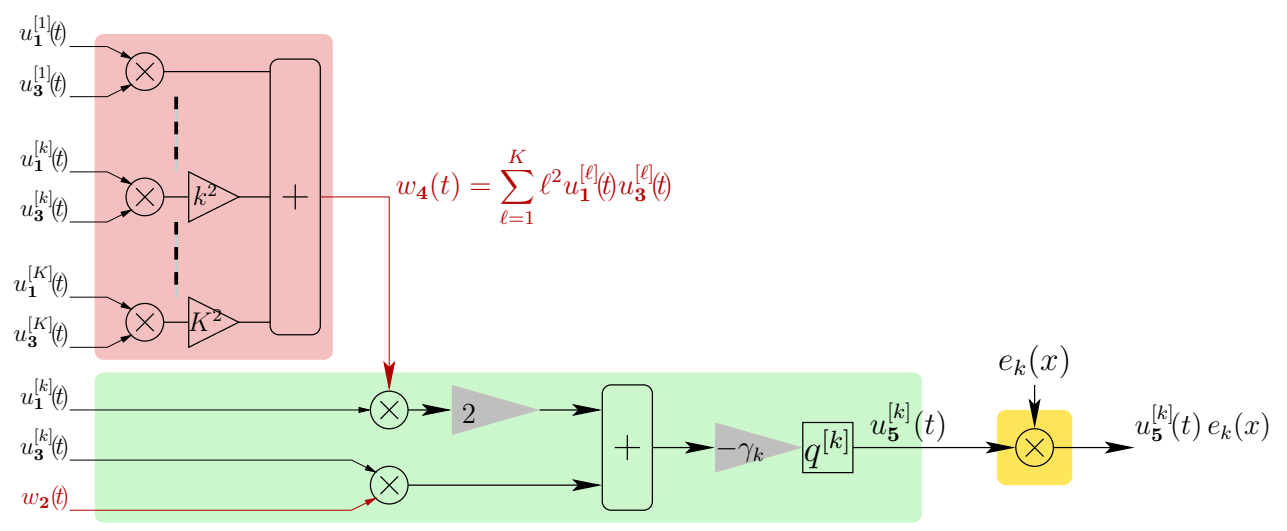

Fig. 15. Realization of $u_{5}^{[k]}$ with its associated modal shape $u_{5}^{[k]}(t) e_{k}(x)$.

are presented in section 7 .

\section{Solution and simulation of (M2)}

Considering a string and impedance type boundary conditions defines a model which can still be solved using Volterra series (with the single input $f$ ). In this case, the kernels depend on the impedance expressions (see [31, chap.4]). In this section, a more general case is considered: that of a string governed by (M2) (see Eqs. (11) to (13)) and driven by three inputs which are a force $f$ inside $\Omega$, a displacement $u_{0}$ at $x=0$ and a displacement $u_{1}$ at $x=1$. This problem makes it necessary to define Volterra series for systems with three inputs, as presented in $\S 5.1$. The solution is derived in $\S 5.2$ from which a simulable structure is identified in $\S 5.3$.

\subsection{Volterra series with three inputs}

For systems with three inputs $\left(e_{\mathrm{I}}, e_{\mathrm{II}}, e_{\mathrm{II}}\right)$ and one output $u$, the definition of Volterra series is generalized as follows: 


$$
\begin{aligned}
u(t)=\sum_{\boldsymbol{m} \in \mathbb{M}} \int_{\mathbb{R}|\boldsymbol{m}|} h_{\boldsymbol{m}}\left(t_{1: \boldsymbol{m}_{\mathrm{I}}}^{\mathrm{I}}, t_{1: \boldsymbol{m}_{\mathbb{I}}}^{\mathrm{II}}, t_{1: \boldsymbol{m}_{\mathrm{II}}}^{\mathrm{II}}\right) e_{\mathrm{I}}\left(t-t_{1}^{\mathrm{I}}\right) \ldots e_{\mathrm{I}}\left(t-t_{\boldsymbol{m}_{\mathrm{I}}}^{\mathrm{I}}\right) \\
\cdot e_{\mathbb{I I}}\left(t-t_{1}^{\mathrm{II}}\right) \ldots e_{\mathrm{II}}\left(t-t_{\boldsymbol{m}_{\mathrm{II}}}^{\mathrm{II}}\right) e_{\mathrm{III}}\left(t-t_{1}^{\mathrm{II}}\right) \ldots e_{\mathrm{II}}\left(t-t_{\boldsymbol{m}_{\mathbb{I I}}}^{\mathrm{II}}\right) \\
\cdot \mathrm{d} t_{1: \boldsymbol{m}_{\mathrm{I}}}^{\mathrm{I}} \mathrm{d} t_{1: \boldsymbol{m}_{\mathbb{I I}}}^{\mathrm{II}} \mathrm{d} t_{1: \boldsymbol{m}_{\mathbb{I I}}^{\mathrm{II}}}
\end{aligned}
$$

where the multi-index $\boldsymbol{m}=\left(\boldsymbol{m}_{\mathrm{I}}, \boldsymbol{m}_{\mathbb{I}}, \boldsymbol{m}_{\mathbb{I I}}\right) \in \mathbb{M}=\mathbb{N}^{3} \backslash\{(0,0,0\}$ details the nonlinearity orders due to each input, while $|\boldsymbol{m}|=\boldsymbol{m}_{\mathrm{I}}+\boldsymbol{m}_{\mathrm{II}}+\boldsymbol{m}_{\mathrm{III}}$ denotes the global nonlinearity order of the kernel $h_{\boldsymbol{m}}$.

Remark 8: For a linear problem, all the kernels $h_{\boldsymbol{m}}$ are zero if $|\boldsymbol{m}| \geq 2$ and, possibly non zero if $|\boldsymbol{m}|=1$. In this case, the three available index $\boldsymbol{m} \in\{(1,0,0) ;(0,1,0) ;(0,0,1)\}$ define the solution as a superposition of three linear contributions: it leads to the standard superposition principle.

Interconnection laws Eqs. (18) to (20) become, respectively, for all $\boldsymbol{m} \in \mathbb{M}$,

$$
\begin{aligned}
& C_{\boldsymbol{m}}\left(s_{\mathbf{1}: \boldsymbol{m}}\right)=A_{\boldsymbol{m}}\left(s_{\mathbf{1}: \boldsymbol{m}}\right)+B_{\boldsymbol{m}}\left(s_{\mathbf{1}: \boldsymbol{m}}\right) \\
& C_{\boldsymbol{m}}\left(s_{\mathbf{1}: \boldsymbol{m}}\right)=\sum_{(\boldsymbol{p}, \boldsymbol{q}) \in \mathbb{M}^{2} \text { s.t. } \boldsymbol{p}+\boldsymbol{q}=\boldsymbol{m}} A_{\boldsymbol{p}}\left(s_{\mathbf{1}: \boldsymbol{p}}\right) B_{\boldsymbol{q}}\left(s_{\boldsymbol{p}+\mathbf{1 :} \boldsymbol{m}}\right) \\
& C_{\boldsymbol{m}}\left(s_{\mathbf{1 : \boldsymbol { m }}}\right)=A_{\boldsymbol{m}}\left(s_{\mathbf{1}: \boldsymbol{m}}\right) B_{1}\left(\widehat{s_{\mathbf{1 :} \boldsymbol{m}}}\right) .
\end{aligned}
$$

with the notations $\mathbf{1}=(1,1,1), \boldsymbol{p}+\boldsymbol{q}=\left(\boldsymbol{p}_{\mathrm{I}}+\boldsymbol{q}_{\mathrm{I}}, \boldsymbol{p}_{\mathrm{II}}+\boldsymbol{q}_{\mathrm{II}}, \boldsymbol{p}_{\mathrm{II}}+\boldsymbol{q}_{\mathrm{II}}\right)$ for $(\boldsymbol{p}, \boldsymbol{q}) \in \mathbb{M}^{2}$ and, if $\boldsymbol{p} \leq \boldsymbol{q}$ (that is $\boldsymbol{p}_{\mathrm{I}} \leq \boldsymbol{q}_{\mathrm{I}}, \boldsymbol{p}_{\mathrm{II}} \leq \boldsymbol{q}_{\mathrm{II}}, \boldsymbol{p}_{\mathrm{III}} \leq \boldsymbol{q}_{\mathrm{III}}$ ),

$$
\begin{aligned}
& \left(s_{\boldsymbol{p}: \boldsymbol{q}}\right)=\left(s_{\boldsymbol{p}_{\mathrm{I}}}^{\mathrm{I}}, s_{\boldsymbol{p}_{\mathrm{I}}+1}^{\mathrm{I}}, \ldots, s_{\boldsymbol{q}_{\mathrm{I}}}^{\mathrm{I}}, s_{\boldsymbol{p}_{\mathrm{II}}}^{\mathrm{II}}, s_{\boldsymbol{p}_{\mathrm{II}}+1}^{\mathrm{II}}, \ldots, s_{\boldsymbol{q}_{\mathrm{II}}}^{\mathrm{II}}, s_{\boldsymbol{p}_{\mathrm{II}}}^{\mathrm{II}}, s_{\boldsymbol{p}_{\mathrm{II}}+1}^{\mathrm{II}}, \ldots, s_{\boldsymbol{q}_{\mathrm{II}}}^{\mathrm{II}}\right), \\
& \widehat{s_{\boldsymbol{p}: \boldsymbol{q}}}=s_{\boldsymbol{p}_{\mathrm{I}}}^{\mathrm{I}}+s_{\boldsymbol{p}_{\mathrm{I}}+1}^{\mathrm{I}}+\ldots+s_{\boldsymbol{q}_{\mathrm{I}}}^{\mathrm{I}}+s_{\boldsymbol{p}_{\mathrm{II}}}^{\mathrm{II}}+s_{\boldsymbol{p}_{\mathrm{II}}+1}^{\mathrm{II}}+\ldots+s_{\boldsymbol{q}_{\mathrm{II}}}^{\mathrm{II}}+s_{\boldsymbol{p}_{\mathrm{II}}}^{\mathrm{III}}+s_{\boldsymbol{p}_{\mathrm{III}}+1}^{\mathrm{II}}+\ldots+s_{\boldsymbol{q}_{\mathrm{II}}}^{\mathrm{II}}(58)
\end{aligned}
$$

\subsection{Derivation of the Volterra kernels for (M2)}

Modelling the displacement $u(x, t)$ using a Volterra series $\left\{h_{m}^{(x)}\right\}_{m \in \mathbb{M}}$ with inputs $e_{\mathrm{I}}(t)=f(t), e_{\mathbb{I I}}(t)=u_{0}(t), e_{\mathrm{III}}(t)=u_{1}(t)$ and translating Eq. (11) into the block-diagram in Fig. 16 allow to derive the following equation through Eqs. (54) to (56): for all $\boldsymbol{m} \in \mathbb{M}$ and $\left(x, s_{\mathbf{1}: \boldsymbol{m}}\right) \in \Omega \times\left(\mathbb{C}_{0}^{+}\right)^{|\boldsymbol{m}|}$,

$$
\left[\Gamma\left(\widehat{s_{\mathbf{1 : m}}}\right)\right]^{2} H_{\boldsymbol{m}}^{(x)}\left(s_{\mathbf{1 : m}}\right)-\frac{\partial^{2} H_{\boldsymbol{m}}^{(x)}\left(s_{\mathbf{1 : m}}\right)}{\partial x^{2}}=\frac{E_{\boldsymbol{m}}^{(x)}\left(s_{\mathbf{1 : m}}\right)}{1+\beta \widehat{s_{\mathbf{1 : m}}}}
$$

with 


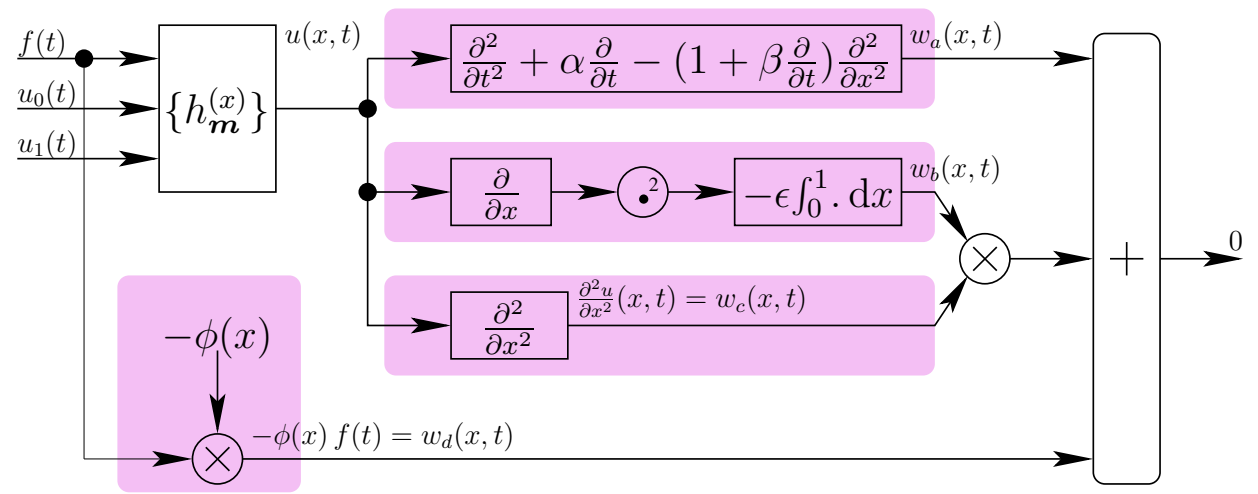

Fig. 16. Block-diagram representing Eq. (11).

$$
\begin{aligned}
& E_{\boldsymbol{m}}^{(x)}\left(s_{1}^{\mathrm{I}}\right)=\phi(x), \quad \text { if } \boldsymbol{m}=(1,0,0), \\
& E_{\boldsymbol{m}}^{(x)}\left(s_{1}{ }^{\mathrm{II}}\right)=0, \quad \text { if } \boldsymbol{m}=(0,1,0), \\
& E_{\boldsymbol{m}}^{(x)}\left(s_{1}^{\mathrm{II}}\right)=0, \quad \text { if } \boldsymbol{m}=(0,0,1), \\
& E_{\boldsymbol{m}}^{(x)}\left(s_{\mathbf{1 : m}}\right)=\epsilon \sum_{\substack{(\boldsymbol{p}, \boldsymbol{q}, \boldsymbol{r}) \in \mathbb{M}^{3} \\
\boldsymbol{p}+\boldsymbol{q}+\boldsymbol{r}=\boldsymbol{m}}} \int_{0}^{1}\left[\frac{\partial H_{\boldsymbol{p}}^{(x)}\left(s_{\mathbf{1}: \boldsymbol{p}}\right)}{\partial x} \frac{\partial H_{\boldsymbol{q}}^{(x)}\left(s_{\boldsymbol{p}+\mathbf{1}: \boldsymbol{p}+\boldsymbol{q}}\right)}{\partial x}\right] \mathrm{d} x \\
& \frac{\partial^{2} H_{r}^{(x)}\left(s_{\boldsymbol{p}+\boldsymbol{q}+\mathbf{1}: \boldsymbol{m}}\right)}{\partial x^{2}} \text { if }|\boldsymbol{m}| \geq 2 .
\end{aligned}
$$

The boundary conditions Eq. (12) translate into, for all $\boldsymbol{m} \in \mathbb{M}$ and $\left(s_{\mathbf{1}: \boldsymbol{m}}\right) \in$ $\left(\mathbb{C}_{0}^{+}\right)^{|\boldsymbol{m}|}$,

$$
\begin{aligned}
& {\left[H_{\boldsymbol{m}}^{(x=0)}\left(s_{1}^{\mathbb{I I}}\right), H_{\boldsymbol{m}}^{(x=1)}\left(s_{1}^{\mathbb{I I}}\right)\right]=[1,0], \quad \text { if } \boldsymbol{m}=(0,1,0),} \\
& {\left[H_{\boldsymbol{m}}^{(x=0)}\left(s_{1}^{\mathrm{II}}\right), H_{\boldsymbol{m}}^{(x=1)}\left(s_{1}^{\mathrm{III}}\right)\right]=[0,1], \quad \text { if } \boldsymbol{m}=(0,0,1),} \\
& {\left[H_{\boldsymbol{m}}^{(x=0)}\left(s_{\mathbf{1}: \boldsymbol{m}}\right), H_{\boldsymbol{m}}^{(x=1)}\left(s_{\mathbf{1}: \boldsymbol{m}}\right)\right]=[0,0], \quad \text { if } \boldsymbol{m}=(1,0,0) \text { or }|\boldsymbol{m}| \geq 2 .(66)}
\end{aligned}
$$

For each $\boldsymbol{m} \in \mathbb{M}$, Eqs. (59) to (66) define a second order linear boundary value problem which can be solved analytically, or through a decomposition on a basis, as in $\S 4.2$. Similarly to (M1), kernels $H_{\boldsymbol{m}}^{(x)}$ are zero for even $|\boldsymbol{m}|$.

More precisely, exact expressions of $H_{\boldsymbol{m}}^{(x)}$ are given by, for all $\boldsymbol{m} \in \mathbb{M},\left(s_{\mathbf{1}: \boldsymbol{m}}\right) \in$ $\left(\mathbb{C}_{0}^{+}\right)^{|\boldsymbol{m}|}$ and using the definitions Eqs. (25) and (28),

$$
\begin{aligned}
H_{\boldsymbol{m}}^{(x)}\left(s_{1}^{\mathrm{II}}\right) & =\sinh \left((1-x) \Gamma\left(s_{1}^{\mathrm{II}}\right)\right) / \sinh \Gamma\left(s_{1}^{\mathrm{II}}\right), \quad \text { if } \boldsymbol{m}=(0,1,0), \\
H_{\boldsymbol{m}}^{(x)}\left(s_{1}^{\mathrm{II}}\right) & =\sinh \left(x \Gamma\left(s_{1}^{\mathrm{II}}\right)\right) / \sinh \Gamma\left(s_{1}^{\mathrm{II}}\right), \quad \text { if } \boldsymbol{m}=(0,0,1), \\
H_{\boldsymbol{m}}^{(x)}\left(s_{\mathbf{1}: \boldsymbol{m}}\right) & =\int_{\Omega} G\left(x, \xi, \widehat{s_{\mathbf{1 : m}}}\right) E_{\boldsymbol{m}}^{(\xi)}\left(s_{\mathbf{1 : m} \boldsymbol{m}}\right) \mathrm{d} \xi, \quad \text { if } \boldsymbol{m}=(1,0,0) \text { or }|\boldsymbol{m}| \geq 2(.69)
\end{aligned}
$$


The functions $e_{k} \in \mathcal{B}$ are the eigen-functions of the problems Eqs. (59) to (66) if $\boldsymbol{m}=\{(1,0,0\}$ or $|\boldsymbol{m}| \geq 2$. The cases $\boldsymbol{m} \in\{(0,1,0) ;(0,0,1)\}$ are associated to two distinct bases of eigen-functions, even if $\mathcal{B}$ still defines an $L^{2}$-orthogonal basis. A decomposition on a $L^{2}$-basis ensures the convergence towards the solution for the quadratic mean but not necessarily at a given location $x$ : this is the so-called Gibbs'effect. It will be precisely the case at $x=0$ and $x=1$, if we choose $\mathcal{B}$ for $\boldsymbol{m} \in\{(0,1,0) ;(0,0,1)\}$. Nevertheless, this choice simplifies the derivation of solutions. It yields the following results, using the definition Eq. (34), for all $\boldsymbol{m} \in \mathbb{M}, k \in \mathbb{N}^{*},\left(s_{\mathbf{1 : m}}\right) \in\left(\mathbb{C}_{0}^{+}\right)^{|\boldsymbol{m}|}$,

$$
\begin{aligned}
& H_{\boldsymbol{m}}^{(x)}\left(s_{\mathbf{1}: \boldsymbol{m}}\right)=\sum_{L^{2}} \sum_{k \in \mathbb{N}^{*}} H_{\boldsymbol{m}}^{[k]}\left(s_{\mathbf{1}: \boldsymbol{m}}\right) e_{k}(x), \\
& H_{\boldsymbol{m}}^{[k]}\left(s_{1}^{\mathrm{I}}\right)=\phi_{k} Q^{[k]}\left(s_{1}^{\mathrm{I}}\right), \quad \text { if } \boldsymbol{m}=(1,0,0), \\
& H_{\boldsymbol{m}}^{[k]}\left(s_{1}^{\mathbb{I}}\right)=\sqrt{2} k \pi\left(1+\beta s_{1}^{\mathbb{I I}}\right) Q^{[k]}\left(s_{1}^{\mathbb{I}}\right), \quad \text { if } \boldsymbol{m}=(0,1,0), \\
& H_{\boldsymbol{m}}^{[k]}\left(s_{1}^{\mathrm{II}}\right)=(-1)^{k+1} \sqrt{2} k \pi\left(1+\beta s_{1}^{\mathrm{II}}\right) Q^{[k]}\left(s_{1}^{\mathrm{II}}\right), \quad \text { if } \boldsymbol{m}=(0,0,1) \text {, } \\
& H_{\boldsymbol{m}}^{[k]}\left(s_{\mathbf{1}: \boldsymbol{m}}\right)=-\epsilon k^{2} \pi^{4} Q^{[k]}\left(\widehat{s_{\mathbf{1 : \boldsymbol { m }}}}\right) \sum_{\substack{(\boldsymbol{p}, \boldsymbol{q}, \boldsymbol{r}) \in \mathbb{M}^{3} \\
\boldsymbol{p}+\boldsymbol{q}+\boldsymbol{r}=\boldsymbol{m}}}\left[\sum_{\ell \in \mathbb{N}^{*}} \ell^{2} H_{\boldsymbol{p}}^{[\ell]}\left(s_{\mathbf{1}: \boldsymbol{p}}\right) H_{\boldsymbol{q}}^{[\ell]}\left(s_{\boldsymbol{p}+\mathbf{1}: \boldsymbol{p}+\boldsymbol{q}}\right)\right] \\
& \cdot H_{\boldsymbol{r}}^{[k]}\left(s_{\boldsymbol{p}+\boldsymbol{q}+\mathbf{1}: \boldsymbol{m}}\right), \quad \text { if }|\boldsymbol{m}| \geq 2 .(74)
\end{aligned}
$$

The combinatorics can be still organized as one sum of elementary terms, as follows.

\section{Theorem 3}

Let $\mathbb{B}_{\boldsymbol{m}}$ be the sets of full ternary trees defined by, for $\boldsymbol{m} \in \mathbb{M}$,

$$
\begin{array}{ll}
\mathbb{B}_{(1,0,0)}=\mathbb{N}^{*} \times\{I\}, \mathbb{B}_{(0,1,0)}=\mathbb{N}^{*} \times\{\mathbb{I}\}, \mathbb{B}_{(0,0,1)}=\mathbb{N}^{*} \times\{\mathbb{I I}\}, & \text { if }|\boldsymbol{m}|=1, \\
\mathbb{B}_{\boldsymbol{m}}=\emptyset, & \text { if }|\boldsymbol{m}| \text { is even, } \\
\mathbb{B}_{\boldsymbol{m}}=\bigcup_{\substack{(\boldsymbol{p}, \boldsymbol{q}, \boldsymbol{r}) \in \mathbb{M}^{3} \\
|\boldsymbol{p}|,|\boldsymbol{q}|,|\boldsymbol{r}| \text { odd } \\
\boldsymbol{p}+\boldsymbol{q}+\boldsymbol{r}=\boldsymbol{m}}}\left\{\left(\mathfrak{b}_{1}, \mathfrak{b}_{2}, \mathfrak{b}_{3}\right) \in \mathbb{B}_{\boldsymbol{p}} \times \mathbb{B}_{\boldsymbol{q}} \times \mathbb{B}_{\boldsymbol{r}} \mid / \mathfrak{k}\left(\mathfrak{b}_{1}\right)=\mathfrak{k}\left(\mathfrak{b}_{2}\right)\right\}, & \text { if }|\boldsymbol{m}| \geq 3 \text { is odd }(77) \\
&
\end{array}
$$

where, for all $\boldsymbol{m} \in \mathbb{M}$ and $\mathfrak{b} \in \mathbb{B}_{\boldsymbol{m}}$, the definition of $\mathfrak{k}(\mathfrak{b})$ is the following: if $|\boldsymbol{m}|=1$ so that $\mathfrak{b}=(k, \iota) \in \mathbb{N}^{*} \times\{\mathrm{I}, \mathbb{I}, \mathbb{I I}\}$, then $\mathfrak{k}(\mathfrak{b})=k$; if $|\boldsymbol{m}| \geq 3$ is odd so that $\mathfrak{b}=\left(\mathfrak{b}_{1}, \mathfrak{b}_{2}, \mathfrak{b}_{3}\right)$, then $\mathfrak{k}(\mathfrak{b})=\mathfrak{k}\left(\mathfrak{b}_{3}\right)$.

Then, for all $\boldsymbol{m} \in \mathbb{M}$, kernels $H_{\boldsymbol{m}}^{(x)}$ are given by, for all $\left(x, s_{\mathbf{1}: \boldsymbol{m}}\right) \in \Omega \times\left(\mathbb{C}_{0}^{+}\right)^{|\boldsymbol{m}|}$,

$$
H_{\boldsymbol{m}}^{(x)}\left(s_{\mathbf{1}: \boldsymbol{m}}\right)=\sum_{\mathfrak{b} \in \mathbb{B}_{\boldsymbol{m}}} H_{\mathfrak{b}}\left(s_{\mathbf{1}: \boldsymbol{m}}\right) e_{\mathfrak{k}(\mathfrak{b})}(x),
$$

where, for all $\mathfrak{b} \in \mathbb{B}_{\boldsymbol{m}}$ (with $|\boldsymbol{m}|$ odd and $\mathfrak{b}=\left(\mathfrak{b}_{1}, \mathfrak{b}_{2}, \mathfrak{b}_{3}\right)$ if $|\boldsymbol{m}| \geq 3$ ), 


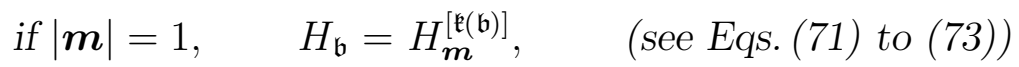

$$
\begin{aligned}
& \text { if }|\boldsymbol{m}| \geq 3, \quad H_{\mathfrak{b}}\left(s_{\mathbf{1}: \boldsymbol{m}}\right)=-\epsilon\left[\mathfrak{k}\left(\mathfrak{b}_{1}\right) \mathfrak{k}\left(\mathfrak{b}_{3}\right) \pi^{2}\right]^{2} Q^{[\mathfrak{k}(\mathfrak{b})]}\left(\widehat{s_{\mathbf{1 : m}}}\right) H_{\mathfrak{b}_{1}}\left(s_{\mathbf{1 : m}\left(\mathfrak{b}_{1}\right)}\right)
\end{aligned}
$$

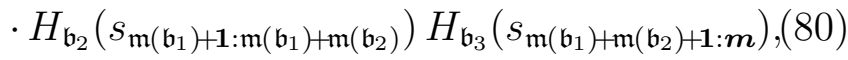

where $\mathfrak{m}(\mathfrak{b})=\left(\boldsymbol{m}_{\mathrm{I}}, \boldsymbol{m}_{\mathbb{I}}, \boldsymbol{m}_{\mathbb{I I}}\right)=\boldsymbol{m}$ counts the number of leaves which are labelled by I, II and III, respectively.

\subsection{Identification of a simulable structure and connection to a bridge}

An identification in Eqs. (70) to (74) for $|\boldsymbol{m}| \leq 3$ and a finite number of modes $(1 \leq k \leq K)$ leads to the structure presented in Fig. 17, which gives an $o(\epsilon)$-approximation of the solution. In this figure, $h_{\boldsymbol{m}}^{[k]}$ are linear filters (see

(S3)

(S4)

(S5)

(S6)

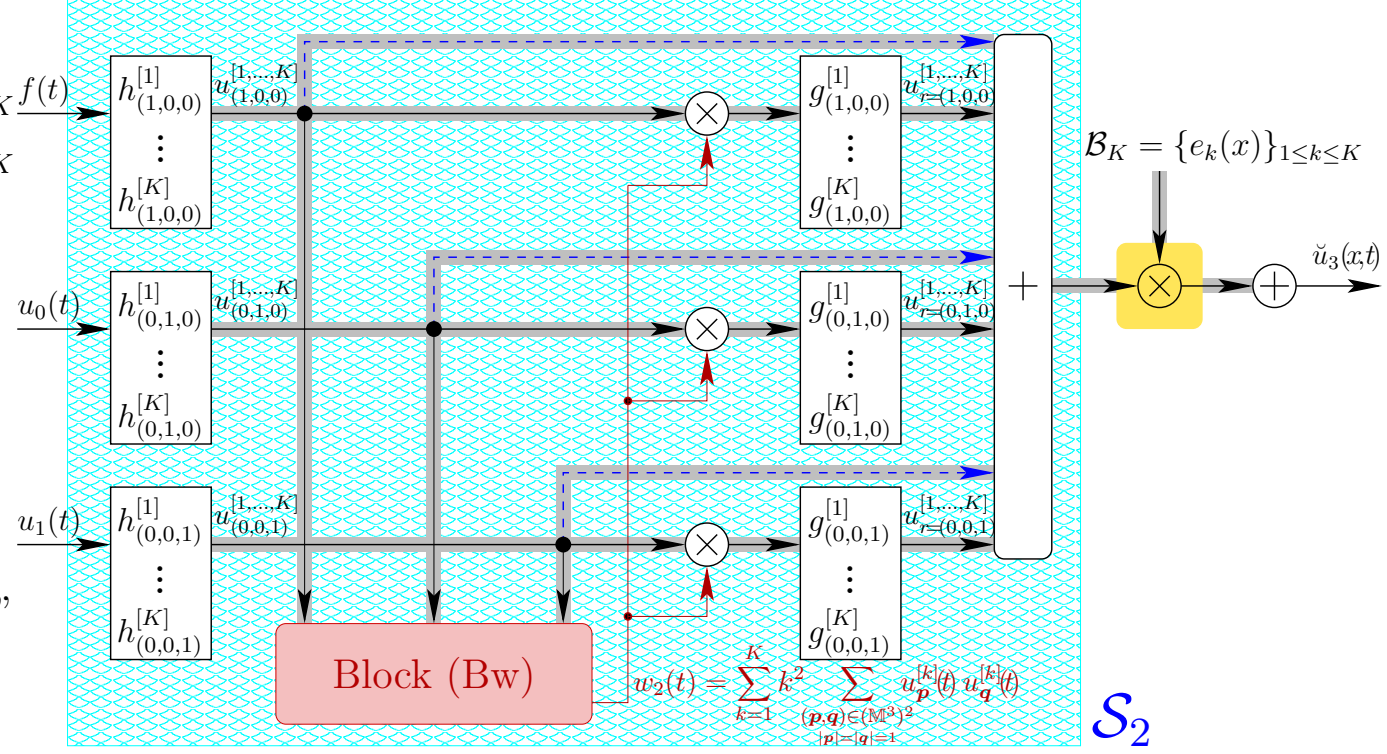

Fig. 17. Block-diagram of an $o(\epsilon)$-simulation of (M2) with $K$ modes: The shaded arrows carry vectors composed of $\mathrm{K}$ signals while the non-shaded ones carry only 1 signal. The first stage (S1) corresponds to three filter-banks of dimension $K$. The first filter-bank is fed by the force $f(t)$, second one by $u_{0}(t)$ and the third one by $u_{1}(t)$. The block $(\mathrm{Bw})$ is described in Fig. 18. The stage (S2) corresponds to the multiplications of a vector signal by a scalar signal in the time domain and (S3) to $3 K$-filters, each filter being fed by one signal. The stage (S4) adds six K-dimensional vectors. Then, outside the structure $\mathcal{S}_{2}$, the stage (S5) multiplies (for each coordinate, separately) the coordinates of two K-dimensional vector, before being added in (S6).

Eqs. (71) to (73)). Their simulation can be performed as for $q^{[k]}$ (see apdx. 9.4) with $\mathbf{C}=\phi_{k}[1,0]$ for $\boldsymbol{m}=(1,0,0)$, with $\mathbf{C}=\sqrt{2} k \pi[1, \beta]$ for $\boldsymbol{m}=(0,1,0)$, and with $\mathbf{C}=(-1)^{k+1} \sqrt{2} k \pi[1, \beta]$ for $\boldsymbol{m}=(0,0,1)$. This is also the case 
for $g_{m}^{[k]}=-\gamma_{k} q^{[k]}$ (see Eq. (52)) which can be performed with $\mathbf{C}=-\gamma_{k}[1,0]$ for $|\boldsymbol{m}|=1$. Thus, all the filters of this structure have the same complexity, namely, $N_{h}^{+}=N_{g}^{+}=4$ and $N_{h}^{\times}=N_{g}^{\times}=5$. The global complexity for a discrete-time realization of this structure with the optimized version of $(\mathrm{Bw})$ presented in Fig. 18(right) is detailed in Table 3.
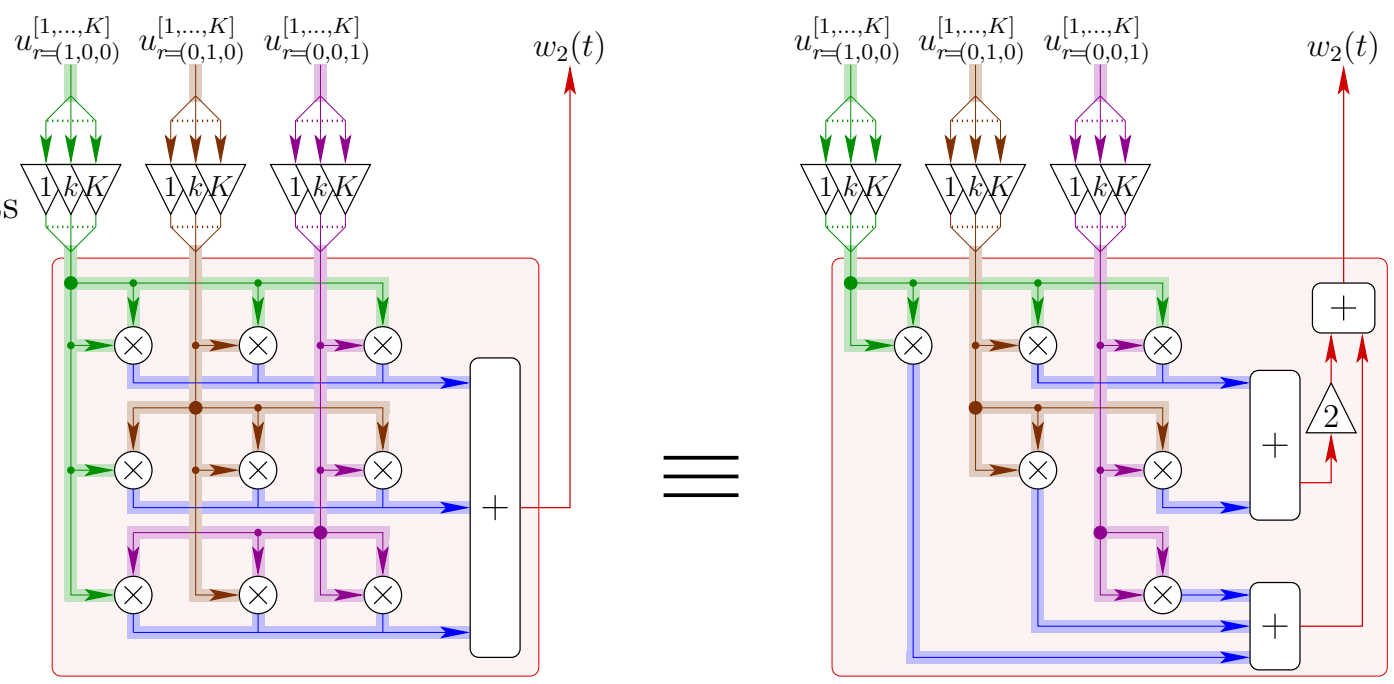

Fig. 18. Detailed block-diagram of $(\mathrm{Bw})$ : the products act on each coordinates separately while sums add all the signals (all coordinates of all vectors).

\begin{tabular}{|l||l|l|}
\cline { 2 - 3 } \multicolumn{1}{l||}{} & linear approx.: $N=1, o\left(\epsilon^{0}\right)$ & third order approx.: $N=3, o\left(\epsilon^{1}\right)$ \\
\hline \hline$N^{+}$ & $K\left(3 N_{h}^{+}+N_{x}+2\right)-N_{x}$ & $K\left(3 N_{h}^{+}+3 N_{g}^{+}+N_{x}+10\right)-N_{x}-1$ \\
\hline$N^{\times}$ & $K\left(3 N_{h}^{\times}+N_{x}\right)$ & $K\left(3 N_{h}^{\times}+3 N_{g}^{\times}+N_{x}+12\right)-2$ \\
\hline$N^{\text {flops }}$ & $K\left(3 N_{h}^{\text {flops }}+2 N_{x}+2\right)-N_{x}$ & $K\left(6 N_{h}^{\text {flops }}+2 N_{x}+22\right)-N_{x}-3$ \\
\hline
\end{tabular}

Table 3

Number of floating point operations to compute $u(x, t)$ for (M2) with $K$ modes, at a given time and at $N_{x}$ distinct locations for (M2): $N_{h}^{+}$denotes the number of sums involved in the simulation of a filter $h_{1}^{[k]}\left(N_{h}^{\times}\right.$for products and $N_{g}^{+}, N_{g}^{\times}$for $\left.g_{1}^{[k]}\right)$.

Remark: Choosing $\mathcal{D}_{K}=\left\{\frac{\partial e_{k}}{\partial x}(x)\right\}_{1 \leq k \leq K}$ in place of $\mathcal{B}_{K}=\left\{e_{k}(x)\right\}_{1 \leq k \leq K}$ computes the output $\frac{\partial u_{3}}{\partial x}(x, t)$. Thus, at $x=0$ or $x=1$, this makes it possible the structure to be connected to other systems at the boundaries, such as a bridge. Note that $\frac{\partial e_{k}}{\partial x}(x)=\sqrt{2} k \pi(-1)^{k x}$ for $x \in\{0,1\}$ and that the convergence holds at these points when $K \rightarrow \infty$. 


\section{Third model}

In this section, the string is supposed to be governed by (M3) (see Eqs. (14) to $(16))$. The resolution is performed similarly to section 4 .

\subsection{Cancelling system}

No equation on the Volterra kernels can be straightforwardly derived from Eq. (14) and interconnection laws Eqs. (18) to (20), because of the square-root in Eq. (14).

Nevertheless, the Volterra kernels $\left\{h_{n}\right\}$ of a nonlinear system $\mathcal{S}$ and those $\left\{\tilde{h}_{n}\right\}$ of $\mathcal{S}_{N}$ (system $\mathcal{S}$ for which the nonlinearity is approximated by its Taylor expansion until order $N$ ) are the same for $n \leq N$.

Thus, to obtain results valid at order $N=3$, a third order expansion of (M3) is sufficient and corresponds to the approximation

$$
\frac{\partial}{\partial x}\left[\frac{\left(1-\frac{2 \epsilon}{\eta}\right) \frac{\partial u}{\partial x}}{\sqrt{1+\eta\left(\frac{\partial u}{\partial x}\right)^{2}}}+\frac{2 \epsilon}{\eta} \frac{\partial u}{\partial x}\right] \approx \frac{\partial}{\partial x}\left[\frac{\partial u}{\partial x}+\zeta\left(\frac{\partial u}{\partial x}\right)^{3}\right]
$$

with $\zeta=\epsilon-\frac{\eta}{2}=2.2710^{-4}$, from which the cancelling system described in Fig. 19 is derived. The kernels $\left\{H_{n}^{(x)}\right\}$ which correspond to this cancelling

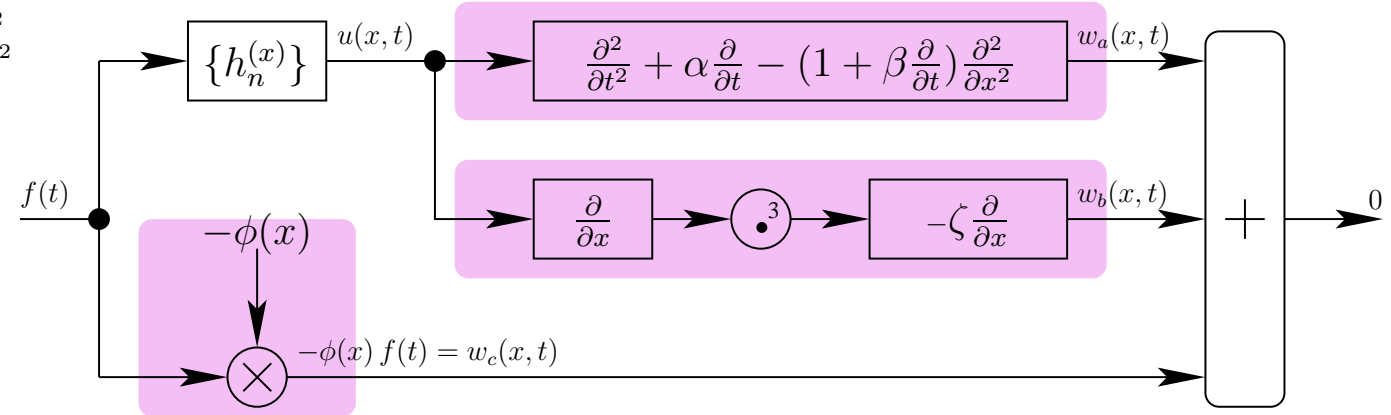

Fig. 19. Cancelling system for the third order approximation of (M3).

system, with the boundary conditions (B1), are solution of Eqs. (22), (23) and Eq. (26). Moreover, if $n \geq 2$, then, for all $\left(x, s_{1: n}\right) \in \Omega \times\left(\mathbb{C}_{0}^{+}\right)^{n}$,

$$
E_{n}^{(x)}\left(s_{1: n}\right)=\zeta \frac{\partial}{\partial x}\left[\sum_{\substack{\left(p_{1: 3}\right) \in\left(\mathbb{N}^{*}\right)^{3} \\ p_{1}+p_{2}+p_{3}=n}} \frac{\partial H_{p_{1}}^{(x)}\left(s_{1: p_{1}}\right)}{\partial x} \frac{\partial H_{p_{2}}^{(x)}\left(s_{p_{1}+1: p_{1}+p_{2}}\right)}{\partial x} \frac{\partial H_{p_{3}}^{(x)}\left(s_{p_{1}+p_{2}+1: n}\right)}{\partial x}\right]
$$


so that analytical solutions can still be derived using Eqs. (27) and (28).

The modal decomposition yields Eqs. (29) to (32) and, if $n \geq 2$, for all $\left(k, s_{1: n}\right) \in \mathbb{N}^{*} \times\left(\mathbb{C}_{0}^{+}\right)^{n}$,

$$
\begin{aligned}
E_{n}^{[k]}\left(s_{1: n}\right) & \left.=-\zeta \sum_{\substack{\left(p_{1: 3}\right) \in\left(\mathbb{N}^{*}\right)^{3} \\
p_{1}+p_{2}+p_{3}=n}} \sum_{\left(k_{1: 3}\right) \in\left(\mathbb{N}^{*}\right)^{3}} \psi_{p_{1}}^{[k]} H_{1: p_{1}}^{\left[k_{1}\right]}\right) H_{p_{2}}^{\left[k_{2}\right]}\left(s_{p_{1}+1: p_{1}+p_{2}}\right) H_{p_{3}}^{\left[k_{3}\right]}\left(s_{p_{1}+p_{2}+1: n}\right),(82) \\
\text { with } \psi_{k_{1: 3}}^{[k]}= & -\left\langle\frac{\partial}{\partial x}\left(\frac{\partial e_{k_{1}}}{\partial x} \frac{\partial e_{k_{2}}}{\partial x} \frac{\partial e_{k_{3}}}{\partial x}\right), e_{k}\right\rangle=\frac{k_{1} k_{2} k_{3} k \pi^{4}}{2} \lambda_{k_{1: 3}}^{[k]} \\
\text { and } \lambda_{k_{1: 3}}^{[k]}= & \sum_{\xi_{2: 3} \in\{-1 ; 1\}^{2}} \delta_{\left|k_{1}+\xi_{2} k_{2}+\xi_{3} k_{3}\right|, k}
\end{aligned}
$$

so that, in Eq. $(82)$, the sum over $\left(k_{1: 3}\right) \in\left(\mathbb{N}^{*}\right)^{3}$ is infinite but sparse.

This combinatorics can be organized as one sum of (non zero) elementary terms, as follows.

\section{Theorem 4}

Let $\mathbb{E}_{n}$ be the sets of full ternary trees defined by, for $n \in \mathbb{N}^{*}$,

$$
\begin{gathered}
\mathbb{E}_{1}=\mathbb{N}^{*}, \\
\mathbb{E}_{n}=\emptyset, \\
\mathbb{E}_{n}=\bigcup_{\substack{\left(p_{1: 3}\right) \in\left(\mathbb{N}^{*}\right)^{3} \\
p_{1: 3} \text { odd } \\
p_{1}+p_{2}+p_{3}=n}}\left\{\mathfrak{e}=\left(\mathfrak{e}_{1}, \mathfrak{e}_{2}, \mathfrak{e}_{3}, \xi_{2: 3}\right) \in \mathbb{E}_{p_{1}} \times \mathbb{E}_{p_{2}} \times \mathbb{E}_{p_{3}} \times\{-1 ; 1\}^{2}\right\}, \\
\text { if } n \geq 3 \text { is odd, (87) }
\end{gathered}
$$

where $\mathfrak{k}(\mathfrak{e})=\mathfrak{e}$ if $\mathfrak{e} \in \mathbb{E}_{1}, \mathfrak{k}(\mathfrak{e})=\left|\mathfrak{k}\left(\mathfrak{e}_{1}\right)+\xi_{2} \mathfrak{k}\left(\mathfrak{e}_{2}\right)+\xi_{3} \mathfrak{k}\left(\mathfrak{e}_{3}\right)\right|$ if $\mathfrak{e}=\left(\mathfrak{e}_{1}, \mathfrak{e}_{2}, \mathfrak{e}_{3}, \xi_{2: 3}\right) \in$ $\mathbb{E}_{n}$ with $n \geq 3$ and $\mathfrak{n}(\mathfrak{e})=n$ counts the number of leaves.

Then, for all $n \in \mathbb{N}^{*}$, kernels $H_{n}^{(x)}$ are given by, for all $\left(x, s_{1: n}\right) \in \Omega \times\left(\mathbb{C}_{0}^{+}\right)^{n}$,

$$
H_{n}^{(x)}\left(s_{1: n}\right)=\sum_{\mathfrak{e} \in \mathbb{E}_{n}} H_{\mathfrak{e}}\left(s_{1: n}\right) e_{\mathfrak{k}(\mathfrak{e})}(x)
$$

where, for all $\mathfrak{e} \in \mathbb{E}_{n}$ (with $n$ odd and $\mathfrak{e}=\left(\mathfrak{e}_{1}, \mathfrak{e}_{2}, \mathfrak{e}_{3}, \xi_{2: 3}\right)$ if $n \geq 3$ ),

$$
\begin{aligned}
& \text { if } n=1, \quad H_{\mathfrak{e}}\left(s_{1}\right)=H_{n}^{[\mathfrak{k}(\mathfrak{e})]}\left(s_{1}\right), \quad(\text { see Eq. } \\
& \text { if } n \geq 3, \quad H_{\mathfrak{e}}\left(s_{1: n}\right)=-\frac{\zeta \mathfrak{k}\left(\mathfrak{e}_{1}\right) \mathfrak{k}\left(\mathfrak{e}_{2}\right) \mathfrak{k}\left(\mathfrak{e}_{3}\right) \mathfrak{k}(\mathfrak{e}) \pi^{4}}{2} Q^{[\mathfrak{k}(\mathfrak{e})]}\left(\widehat{s_{1: n}}\right) H_{\mathfrak{e}_{1}}\left(s_{1: \mathfrak{n}\left(\mathfrak{e}_{1}\right)}\right) \\
& \cdot H_{\mathfrak{e}_{2}}\left(s_{\mathfrak{n}\left(\mathfrak{e}_{1}\right)+1: \mathfrak{n}\left(\mathfrak{e}_{1}\right)+\mathfrak{n}\left(\mathfrak{e}_{2}\right)}\right) H_{\mathfrak{e}_{3}}\left(s_{\mathfrak{n}\left(\mathfrak{e}_{1}\right)+\mathfrak{n}\left(\mathfrak{e}_{2}\right)+1: n}\right) .
\end{aligned}
$$


Another non sparse reorganization of Eq. (82) is given by, if $n \geq 2$, for all $\left(k, s_{1: n}\right) \in \mathbb{N}^{*} \times\left(\mathbb{C}_{0}^{+}\right)^{n}$,

$$
\begin{array}{r}
E_{n}^{[k]}\left(s_{1: n}\right)=-\chi_{k} \sum_{\substack{\left(p_{1: 3}\right) \in\left(\mathbb{N}^{*}\right)^{3} \\
p_{1}+p_{2}+p_{3}=n}} \sum_{\substack{\left.k_{1: 3}\right) \in \mathbb{K}_{k}^{3}\\
}} k_{1} k_{2} k_{3} \lambda_{k_{1: 3}}^{[k]} H_{p_{1}}^{\left[k_{1}\right]}\left(s_{1: p_{1}}\right) \\
H_{p_{2}}^{\left[k_{2}\right]}\left(s_{p_{1}+1: p_{1}+p_{2}}\right) H_{p_{3}}^{\left[k_{3}\right]}\left(s_{p_{1}+p_{2}+1: n}\right),
\end{array}
$$

where $\chi_{k}=\frac{\zeta k \pi^{4}}{2}, \mathbb{K}_{k}^{3}=\left\{\left(k_{1: 3}\right) \in\left(\mathbb{N}^{*}\right)^{3}\left|\exists\left(\xi_{2}, \xi_{3}\right) \in\{-1 ;+1\}\right| \mid k_{1}+\xi_{2} k_{2}+\right.$ $\left.\xi_{3} k_{3} \mid=k\right\}$ is illustrated in Fig. 20, and the weights $\lambda_{k_{1: 3}}^{[k]}$ are detailed in Table 4 . Thus, for a finite number $K$ of modes $(1 \leq k \leq K)$, identifying linear and

(a)

(1)

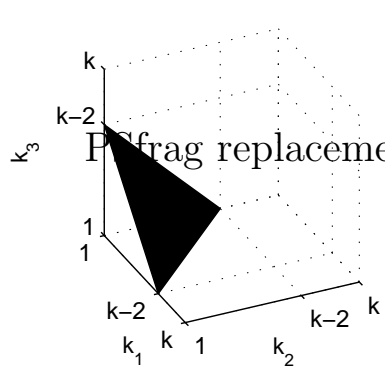

(2) (b)
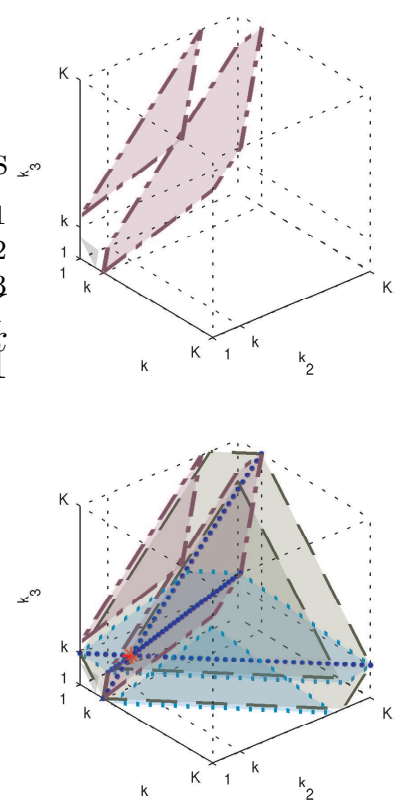

(C)
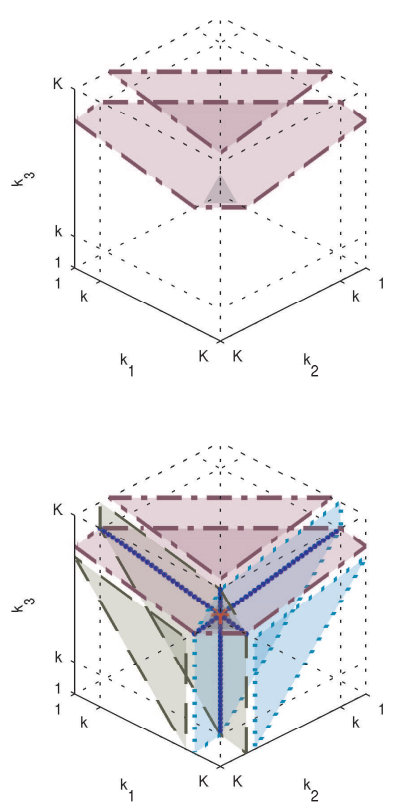

Fig. 20. The set $\mathbb{K}_{k}^{3}$ is composed of triplets of integers $\left(k_{1: 3}\right)$ which belong to: a triangle if $\left(\xi_{2: 3}\right)=(1,1)$ (see (1a)); a couple of semi-infinite parts of parallel planes if $\left(\xi_{2: 3}\right)=(1,-1)($ see $(\mathbf{1 b}, \mathbf{1} \mathbf{c}))$; the cases $\left(\xi_{2: 3}\right)=(-1,1)$ and $\left(\xi_{2: 3}\right)=(-1,-1)$ are symmetric versions of the previous case. All the contributions are gathered in $(\mathbf{2 b}, \mathbf{2 c})$. Parts which are not intersected correspond to a weight $\lambda_{k_{1}: 3}^{[k]}=1$, intersections of 2 planes (blue points) to $\lambda_{k_{1: 3}}^{[k]}=2$, the intersection of three planes (one red point, $\left.k_{1}=k_{2}=k_{3}=k\right)$ to $\lambda_{k_{1: 3}}^{[k]}=3$.

third order kernels from Eq. (91) rather than Eq. (33) leads to the structure in Fig. 21 (to be compared to Fig. 14). In this structure, each output $v_{3}^{[k]}(t)$ of 


\begin{tabular}{|c|c|c|c|c|}
\hline Cases & $\lambda_{k_{1: 3}^{[k]}}$ & Subcases & $C_{k_{1: 3}}$ & $\tilde{\lambda}_{k_{1: 3}^{[k]}}$ \\
\hline (1): $k_{1}=k_{2}=k_{3}=k$ & 3 & none & 1 & 3 \\
\hline $\begin{array}{l}\text { (2): } k_{1}=k_{2} \neq k \text { and } k_{3}=k \\
\text { (and the two permutations) }\end{array}$ & 2 & none & 3 & 6 \\
\hline \multirow[t]{3}{*}{ (3): Other cases, if $\left(k_{1: 3}\right) \in \mathbb{K}_{k}^{3}$} & \multirow[t]{3}{*}{1} & (a): $k_{1}=k_{2}=k_{3} \neq k$ & 1 & 1 \\
\hline & & $\begin{array}{l}\text { (b): } k_{1}=k_{2} \neq k_{3} \neq k \\
\text { (and the two permutat.) }\end{array}$ & 3 & 3 \\
\hline & & (c): $k_{1} \neq k_{2} \neq k_{3} \neq k_{1}$ & 6 & 6 \\
\hline (4): Other cases, if $\left(k_{1: 3}\right) \notin \mathbb{K}_{k}^{3}$ & 0 & $\times$ & $\times$ & 0 \\
\hline
\end{tabular}

Table 4

Detailed weights $\lambda_{k_{1: 3}}^{[k]}$ and $\tilde{\lambda}_{k_{1: 3}}^{[k]}$.

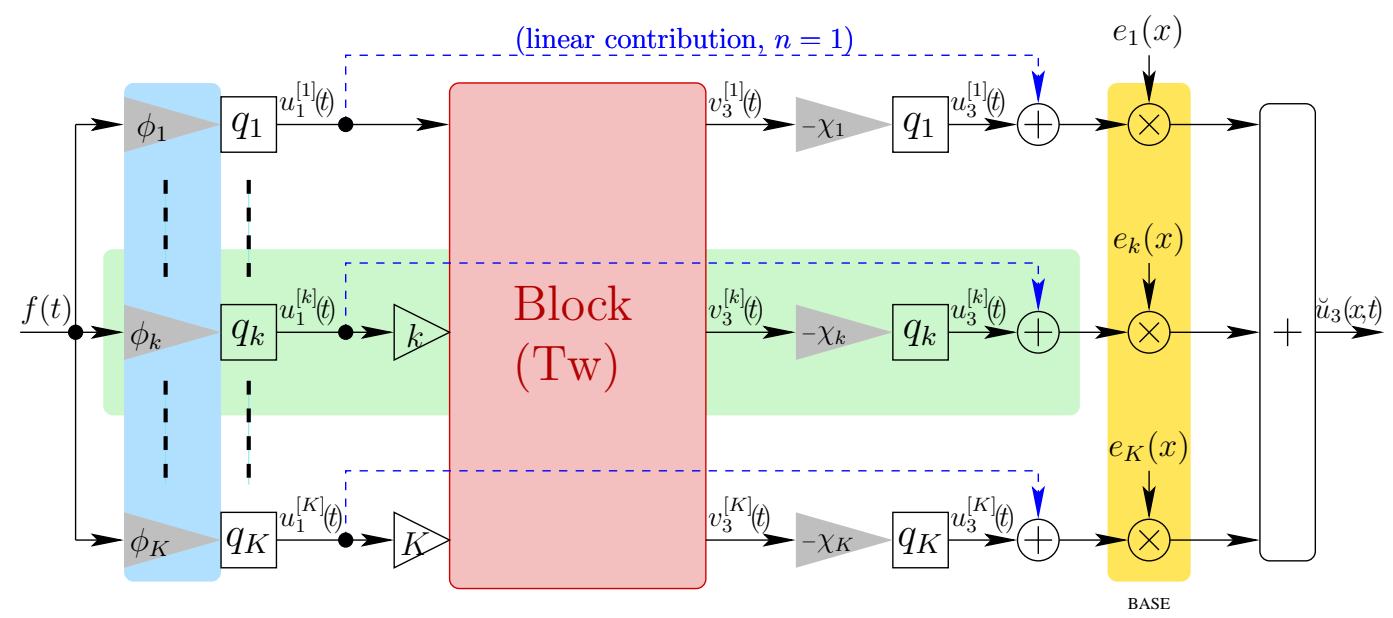

Fig. 21. Block-diagram of a $o\left(\epsilon_{3}\right)$-simulation of (M3) with $K$ modes. Each output $v_{3}^{[k]}(t)$ of the block $(\mathrm{Tw})$ is a linear combination of products of triplets $\left(u_{k_{1}}^{[1]}(t) u_{k_{2}}^{[1]}(t) u_{k_{3}}^{[1]}(t)\right)$ (see Eq. $\left.(92)\right)$.

the block (Tw) is given by, for all $k \in \mathbb{N}^{*}$ and $t \in \mathbb{R}^{+}$,

$$
v_{3}^{[k]}(t)=\sum_{\left(k_{1: 3}\right) \in \mathbb{K}_{k}^{3} \cap[1, K]_{\mathbb{N}}^{3}} \lambda_{k_{1: 3}}^{[k]} k_{1} k_{2} k_{3} u_{k_{1}}^{[1]}(t) u_{k_{2}}^{[1]}(t) u_{k_{3}}^{[1]}(t)
$$

for which the combinatorics can be significantly reduced (about a factor 4) thanks to symmetric considerations. Indeed, similarly to Fig. 18 for the block (Bw), only the triangular superior part $\tilde{\mathbb{K}}_{k}^{3}$ of $\mathbb{K}_{k}^{3}$ can be preserved (see Fig. 22), with adapted weights $\tilde{\lambda}_{k_{1: 3}}^{[k]}$ (see Table 4 ), as follows: 


$$
\begin{aligned}
\tilde{\mathbb{K}}_{k}^{3} & =\mathbb{K}_{k}^{3} \cap\left\{\left(k_{1: 3}\right) \in[1, K]_{\mathbb{N}}^{3} \mid k_{1} \leq k_{2} \leq k_{3}\right\}, \\
\tilde{\lambda}_{k_{1: 3}}^{[k]} & =C_{k_{1: 3}} \lambda_{k_{1: 3}}^{[k]} \\
\text { with } C_{k_{1: 3}} & =\operatorname{card}\left\{\left(k_{1}, k_{2}, k_{3}\right) ;\left(k_{1}, k_{3}, k_{2}\right) ;\left(k_{2}, k_{1}, k_{3}\right) ;\right. \\
& \left.\quad\left(k_{3}, k_{1}, k_{2}\right) ;\left(k_{2}, k_{3}, k_{1}\right) ;\left(k_{3}, k_{2}, k_{1}\right)\right\} .
\end{aligned}
$$

The simulation of the structure in Fig.21 involves exactly the same filters as
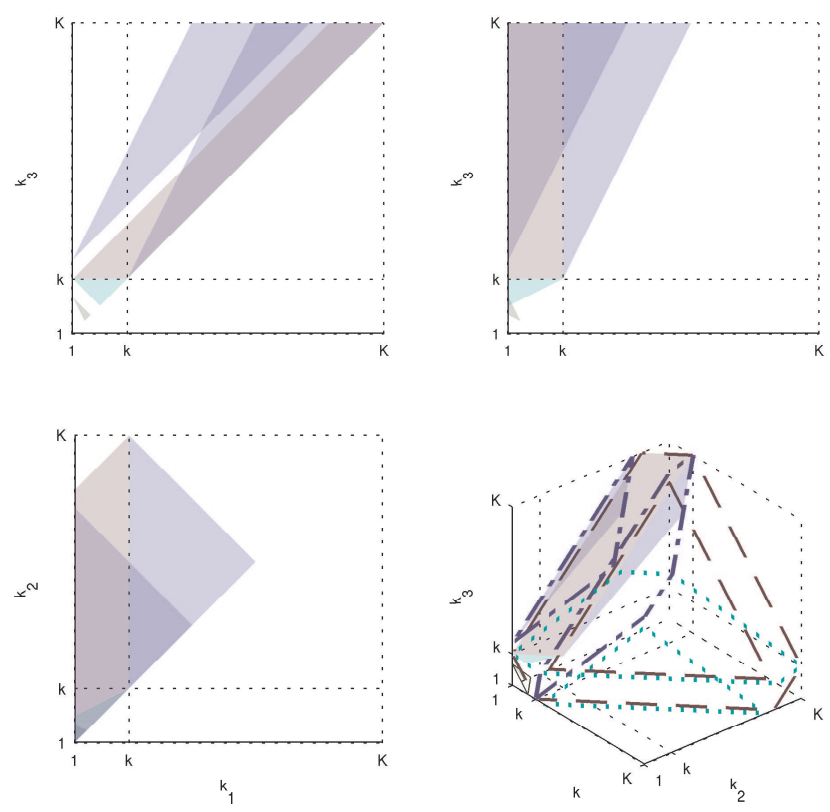

Fig. 22. Views of the set $\tilde{\mathbb{K}}_{k}^{3}$.

in Fig.14. The only difference is the block ( $\mathrm{Tw})$, the simulation of which is performed using Eq. (92), for each mode $k$ and at each time.

\section{Sound synthesis}

In this section, we propose to simulate models (M1) and (M3) for the following parameters:

- the geometry and the physical constants are those given in Table 1.

- the excitation $\phi(x) f(t)$ is defined by, for $x \in] 0, L\left[\right.$ and $t \in \mathbb{R}_{+}^{*}$

$$
\begin{aligned}
\phi(x) & =\phi_{\max } \cos \left(\pi \frac{x-x_{0}}{\ell}\right) 1_{\left[x_{0}-\frac{\ell}{2}, x_{0}+\frac{\ell}{2}\right]}(x), \\
f(t) & =F_{\max } \frac{t}{T} 1_{[0, T]}(t),
\end{aligned}
$$

where $x_{0}=0.63 \mathrm{~m}, \ell=0.072 \mathrm{~m}\left(\tilde{x}_{0}=x_{0} / L=0.35, \tilde{\ell}=\ell / L=0.04\right.$ so that $4 \%$ of string is plucked), $\phi_{\max }=\pi /(2 \rho A \ell)=395.7 \mathrm{Kg}^{-1}$ so that Eq. (1) is satisfied 
$\left(\tilde{\phi}_{\max }=\pi /(2 \tilde{\ell})=39.27\right), T=10 \mathrm{~ms}(\tilde{T}=1.1)$. Several Forces $F_{\max }$ are used to investigate the nonlinear effects: $F_{\max }^{1}=5 \mathrm{~N}, F_{\max }^{2}=20 \mathrm{~N}, F_{\max }^{3}=40 \mathrm{~N}$, and $F_{\max }^{4}=160 \mathrm{~N}\left(\tilde{F}_{\max }^{1}=2.8, \tilde{F}_{\max }^{2}=11.1, \tilde{F}_{\max }^{3}=22.2, \tilde{F}_{\max }^{4}=88.8\right)$.

- the sampling frequency is $f_{s}=44100 \mathrm{~Hz}\left(\tilde{f}_{s}=401\right)$, the number of modes is $K=20$, the order of approximation is $N=3$ in sec.7.1, and $N=5$ in sec. 7.2 .

For these data, the resonance frequencies $f_{k}=\frac{c}{L} \sqrt{k^{2} \pi^{2}-\left(\alpha+\beta k^{2} \pi^{2}\right)^{2}} /(2 \pi)$ of the band-pass filters with kernel $q_{1}^{[k]}$ (see Eqs. (34) and (35) grow from $f_{1} \approx 55 \mathrm{~Hz}$ to $f_{K} \approx 1100 \mathrm{~Hz}$. The aliasing due to the nonlinearities is rejected since $N \leq N_{\max }=\frac{f_{s}}{2 f_{K}}\left(1+\frac{1}{Q_{K}}\right)^{-1} \approx 20$ (see Eq. (53)).

\subsection{Third order approximations $(N=3)$}

Figs. 23 to 26 present third order approximations $\breve{u}_{3}(x, t)$ of the (dimensional) displacement observed at $x=0.57 L \approx 1 \mathrm{~m}$.
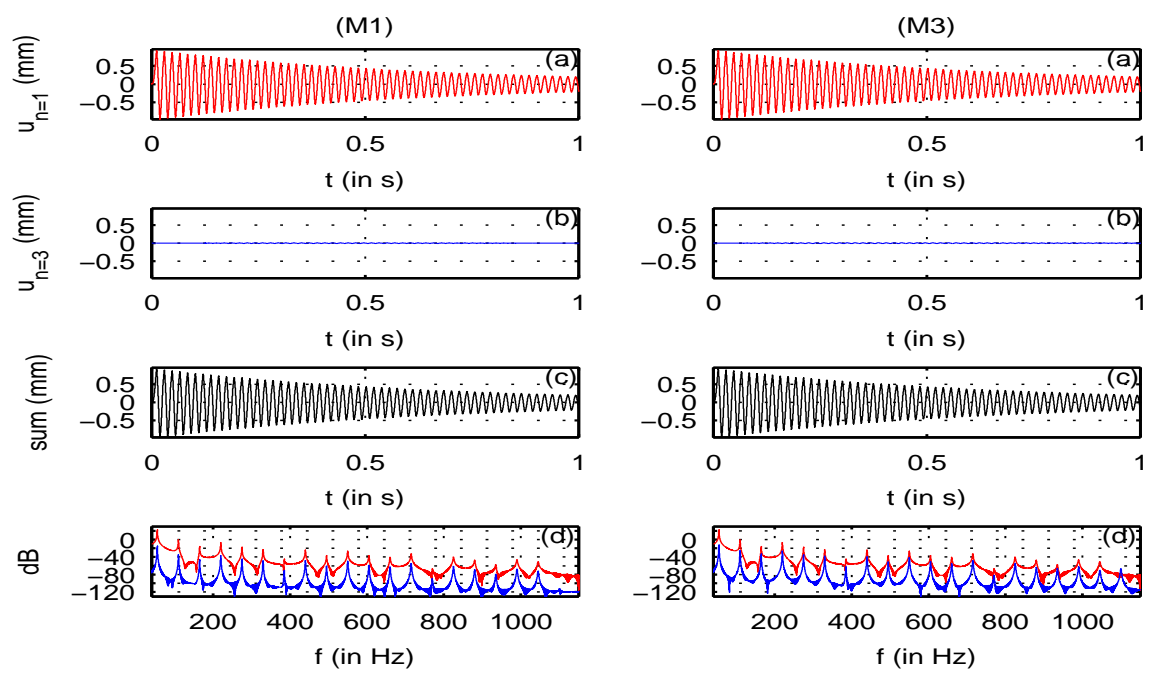

Fig. 23. Simulation of (M1) (left) and (M3) (right) for the same excitation with magnitude $F_{\max }=5 \mathrm{~N}$. The output $u$ is observed at $x=0.57 L \approx 1 \mathrm{~m}$. From top to bottom: (a) linear response, (b) output of order 3 alone, (c) sum of the linear and of the nonlinear contributions, and (d) magnitude of the spectrum of (a) and (b).

For the lowest excitation $F_{\max }^{1}=5 \mathrm{~N}$ (Fig. 23), the nonlinear contribution is negligible for both models (M1) and (M3): the spectrum of $u_{3}$ is approximately $50 \mathrm{~dB}$ lower than that of the linear displacement $u_{1}$. In the time domain, the maximal value of contributions $u_{1}$ and $u_{3}$ are $\max _{t}\left|u_{1}\right| \approx 0.9 \mathrm{~mm}$ and $\max _{t}\left|u_{3}\right|<0.012 \mathrm{~mm}$, respectively. In this case, considering nonlinear models and using Volterra series are needless. As a matter of fact, (M1) and (M3) 

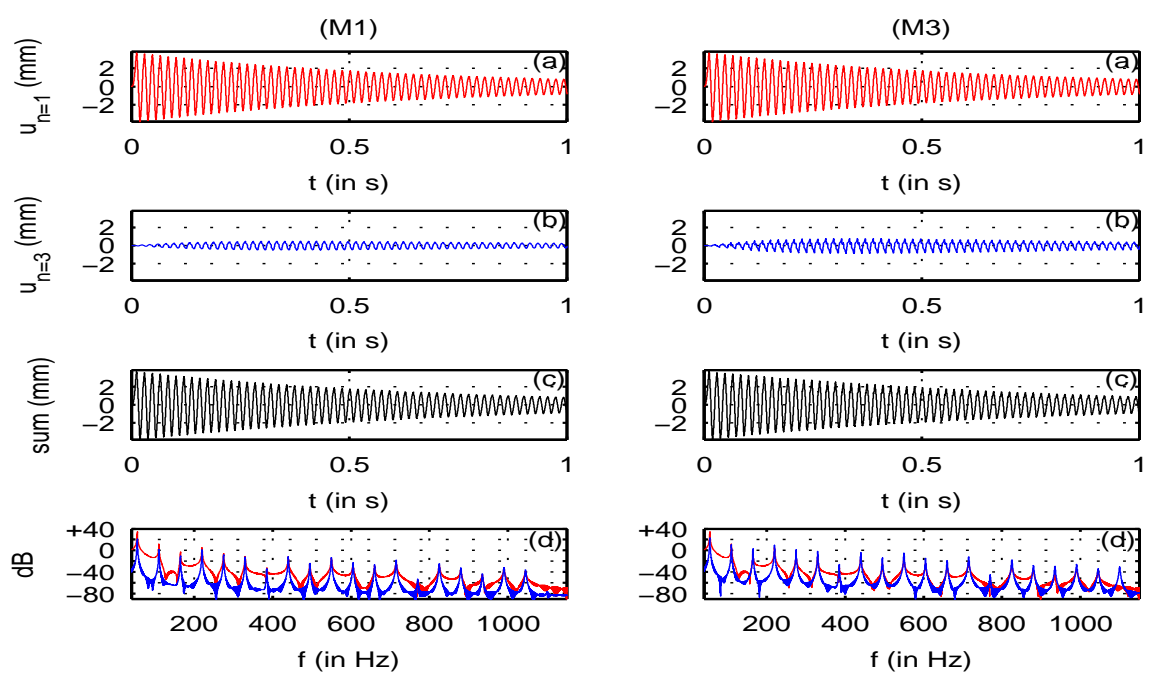

Fig. 24. Idem Fig. 23 with $F_{\max }^{2}=10 \mathrm{~N}$.
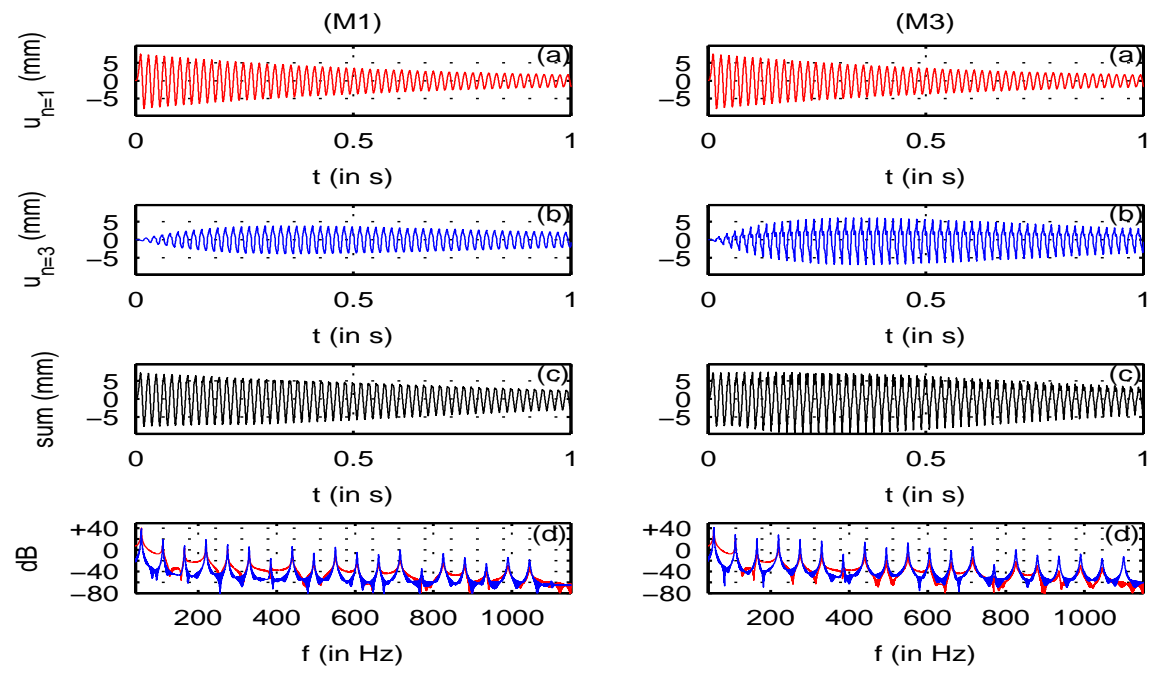

Fig. 25. Idem Fig. 23 with $F_{\max }^{3}=40 \mathrm{~N}$.

become equivalent since, precisely, their linear kernels $h_{1}^{(x)}$ are the same.

For $F_{\max }^{2}=20 \mathrm{~N}$ (Fig. 24), the nonlinear contributions $u_{3}$ begin to be significantly activated and have similar shapes for both models ((M1) and (M3)). Nevertheless, this activation is slightly more perceptible for (M3) than for (M1): while $\max _{t}\left|u_{1}\right| \approx 3.7 \mathrm{~mm}$ (for both models), $\max _{t}\left|u_{3}\right| \approx 0.5 \mathrm{~mm}$ for (M1) and $\max _{t}\left|u_{3}\right| \approx 0.8 \mathrm{~mm}$ for (M3). From a qualitative point of view, it can be observed on the spectra that the eigen-frequencies are the same for the linear and the nonlinear contributions. This is because the order of the nonlinearity is odd.

For the case $F_{\max }^{3}=40 \mathrm{~N}$ (Fig. 25), contributions $u_{1}$ and $u_{3}$ have similar magnitudes (in the time domain as well as the frequency domain): $\max _{t}\left|u_{1}\right| \approx$ 

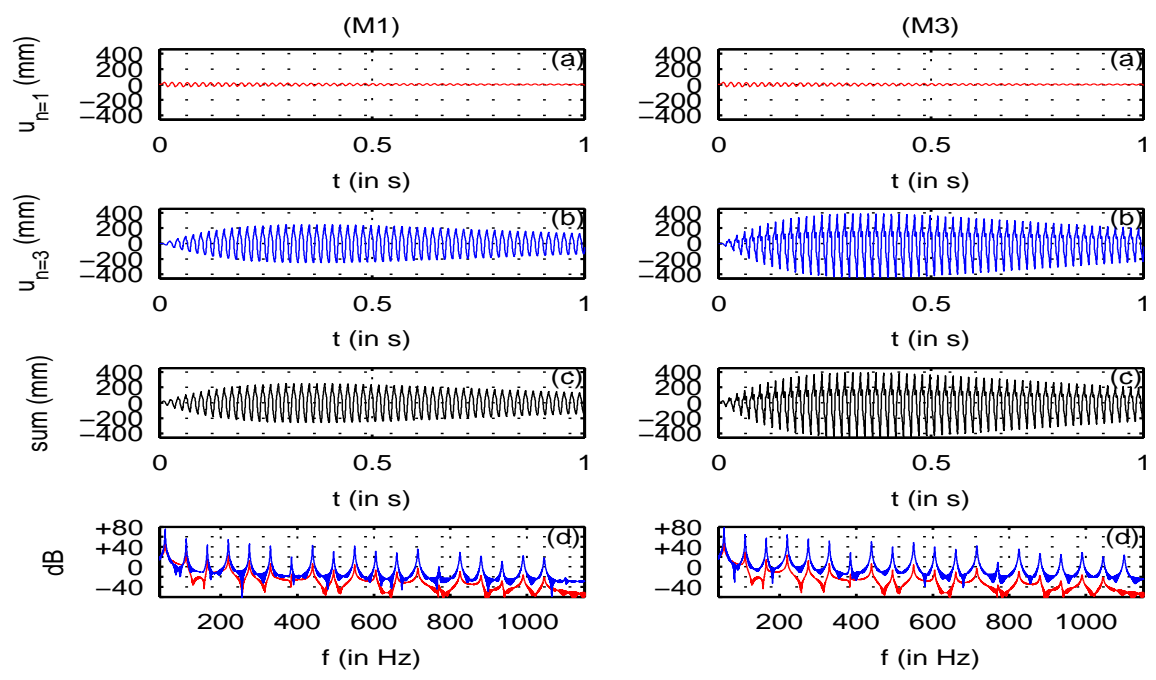

Fig. 26. Idem Fig. 23 with $F_{\max }^{4}=160 \mathrm{~N}$.

$7.4 \mathrm{~mm}$ (for both models), $\max _{t}\left|u_{3}\right| \approx 3.84 \mathrm{~mm}$ for (M1) and $\max _{t}\left|u_{3}\right| \approx$ $6.04 \mathrm{~mm}$ for (M3). In practice, this case can be viewed as the limit of the $o(\epsilon)$-approximation validity, and more generally, of using truncated Volterra series.

Finally, for $F_{\text {max }}^{4}=160 \mathrm{~N}$ (Fig. 26), the nonlinear contribution $u_{3}$ are greater than the linear one for both models: $\max _{t}\left|u_{1}\right| \approx 29.5 \mathrm{~mm}$ (for both models), $\max _{t}\left|u_{3}\right| \approx 245.7 \mathrm{~mm}$ for (M1) and $\max _{t}\left|u_{3}\right| \approx 386.6 \mathrm{~mm}$ for (M3). The approximation is not valid anymore.

In practice, such simulations make the estimation of the "operating range" accessible. But, a more rigorous way would consist of deriving the radius of convergence of the series and a bound of the error due to the truncature of the series. Such results can be found for ordinary differential equations with a quadratic nonlinearity [27] but must be generalized to nonlinear partial differential equations.

Estimations of the fundamental frequency [33] make some time-variations appear when the nonlinear contributions are significant. But more discernible is the transfer of energy from the low frequencies to the higher modes through the nonlinear contributions: in Fig. 25, the linear and the nonlinear contributions have similar magnitudes for the first modes (spectrum), while the nonlinear part has more energy than the linear one in the medium and high frequency range. This is clearly perceptible in the synthesized sounds which are more brilliant at the beginning (before becoming significantly damped). All the more, the transfer of energy is responsible for transients because of its progressive activation (see Figs. 24 and 25 for $0 \leq t \leq 0.2 \mathrm{~s}$ ).

Thus, on a specific given "operating range", Volterra series can be an inter- 
esting alternative to methods such as nonlinear modes which are limited to particular excitations, or finite difference methods which require the solving of the model overall the domain $\Omega$. With no space discretization, Volterra series can give interesting results at reasonable orders.

\subsection{Higher order approximations $(N=5)$}

Figs. 27 to 28 detail the contributions at order 5 for the model (M1) and complete Figs. 23 to 26.
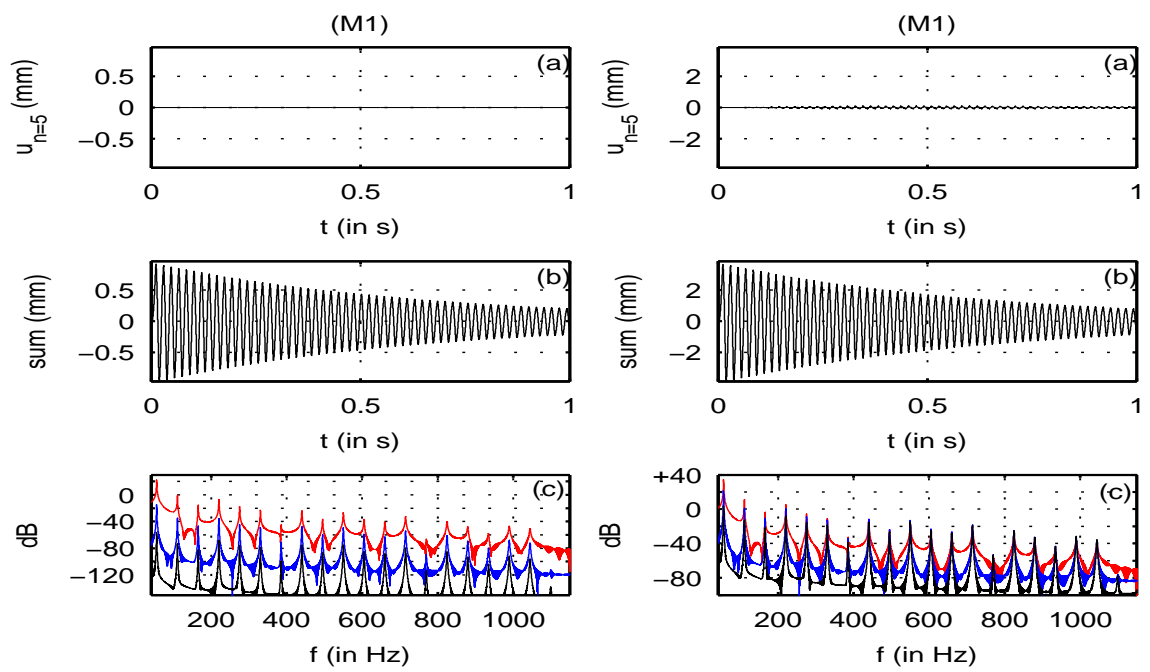

Fig. 27. Simulation of (M1) for $F_{\max }^{1}=5 \mathrm{~N}$ and $F_{\max }^{2}=20 \mathrm{~N}$. From top to bottom: (a) output of order 5 alone, (b) sum of the contributions up to order 5 , and (c) magnitude of the spectrum (order 1,3 , and 5 ).

These results corroborate the observations given for order $n=3$ : the contribution at order 5 is negligible for $F_{\max }^{1}\left(\max _{t}\left|u_{5}\right| \approx 9.610^{-5} \mathrm{~mm}\right)$, lower than that of order 3 for $F_{\max }^{2}\left(\max _{t}\left|u_{5}\right| \approx 0.01 \mathrm{~mm}\right)$, similar to that of order 3 for $F_{\max }^{3}\left(\max _{t}\left|u_{5}\right| \approx 3.1 \mathrm{~mm}\right)$, and greater than that of order 3 for $F_{\max }^{4}$ $\left(\max _{t}\left|u_{5}\right| \approx 3226 \mathrm{~mm}\right)$.

Figs. 27 and 28 show that the nonlinear contribution at order 5 requires higher excitation force to appear that at order 3. The transfer of energy from low to high frequencies is more important. In the time domain, this contribution increases more slowly, thus enhancing the "brillance" effect at the beginning of the sound. 

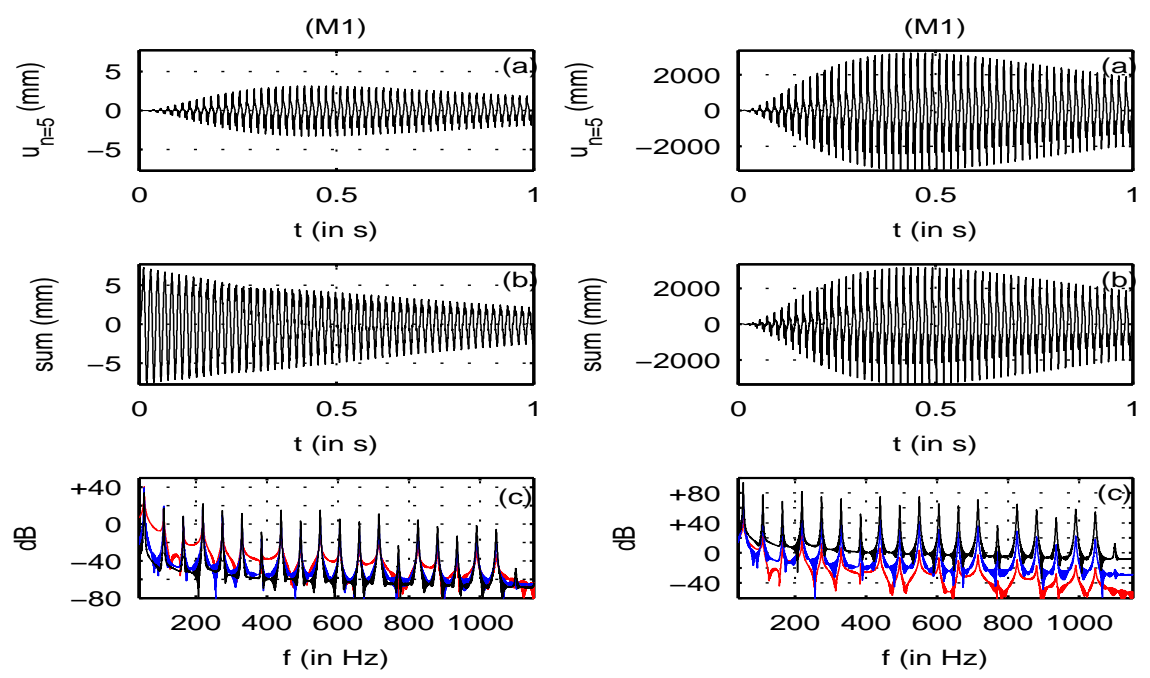

Fig. 28. Idem Fig. 27 for $F_{\max }^{3}=40 \mathrm{~N}$ and $F_{\max }^{4}=160 \mathrm{~N}$.

\section{Conclusion and perspectives}

This work has presented an application of Volterra series to simulate nonlinear vibrations of a string. The method proves to be relevant for the (possibly realtime) sound synthesis of some string instruments for which excitations can be quite large. It has been illustrated with three models with one or several located excitations, and global or local nonlinearities. Systematic identifications have allowed the building of structures which give rise to original physical interpretations, and from which efficient algorithms have been deduced. Indeed, these involve only elementary floating point operations (sums and products without infinite loops), the number of which can be precisely estimated.

Nevertheless, the convergence of the series and the estimation of a bound of the error due to truncation are still difficult to tackle. Some future work could focus on this point. Moreover, 3D-string models which take into account coupled waves can be necessary for the naturalness of the sound synthesis. Hence, the study of more accurate models of strings and the analysis of the perception of nonlinear effects by the listener will be part of following works. More generally, using Volterra series could be applied to other physical models (bi- or tri-dimensional), and some other generalizations could be carried out, e.g. extensions to the finite elements method.

\section{Acknowledgments}

The authors thank the "Agence Nationale de la Recherche" for supporting this research through the "projet blanc ANR-GIP CONSONNES". 


\section{$9 \quad$ Appendix}

9.1 Green function for the linearized problem: (M1) with $\epsilon=0$

Eq. (22) can be written

$\forall\left(x, s_{1: n}\right) \in \Omega \times\left(\mathbb{C}_{0}^{+}\right)^{n}, \frac{\partial \mathbf{X}_{n}^{(x)}\left(s_{1: n}\right)}{\partial x}=\mathbf{A}\left(\widehat{s_{1: n}}\right) \mathbf{X}_{n}^{(x)}\left(s_{1: n}\right)+\mathbf{B}\left(\widehat{s_{1: n}}\right) E_{n}^{(x)}\left(s_{1: n}\right)$,

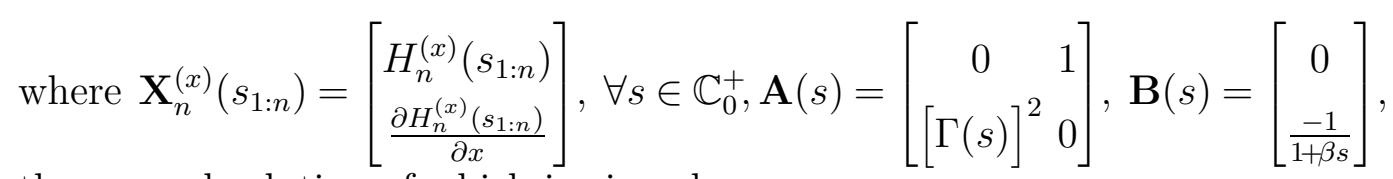
the general solution of which is given by

$$
\mathbf{X}_{n}^{(x)}\left(s_{1: n}\right)=\int_{0}^{x} \mathrm{e}^{(\xi-x) \mathbf{A}\left(\widehat{s_{1: n}}\right)} \mathbf{B}\left(\widehat{s_{1: n}}\right) E_{n}^{(\xi)}\left(s_{1: n}\right) \mathrm{d} \xi+\mathrm{e}^{x} \widehat{\mathbf{A}\left(\widehat{s_{1: n}}\right)} \mathbf{X}_{n}^{(0)}\left(s_{1: n}\right)
$$

where $\mathrm{e}^{x \mathbf{A}(s)}=\left[\begin{array}{ll}\mathbf{E}_{11}(x, s) & \mathbf{E}_{12}(x, s) \\ \mathbf{E}_{21}(x, s) & \mathbf{E}_{22}(x, s)\end{array}\right]=\left[\begin{array}{cc}\cosh (x \Gamma(s)) & \sinh (x \Gamma(s)) / \Gamma(s) \\ \sinh (x \Gamma(s)) \Gamma(s) & \cosh (x \Gamma(s))\end{array}\right]$.

From the boundary conditions Eq. $(26),[1,0] \mathbf{X}_{n}^{(x)}\left(s_{1: n}\right)=0$ for $x \in\{0 ; 1\}$ which implies, from Eq. (99), that

$$
\forall\left(s_{1: n}\right) \in\left(\mathbb{C}_{0}^{+}\right)^{n}, \quad \frac{\partial H_{n}^{(0)}\left(s_{1: n}\right)}{\partial x}=\int_{0}^{1} F\left(\xi, \widehat{s_{1: n}}\right) E_{n}^{(\xi)}\left(s_{1: n}\right) \mathrm{d} \xi
$$

with $F(x, s)=\frac{\mathbf{E}_{12}(x-1, s)}{(1+\beta s) \mathbf{E}_{12}(1, s)}$. Hence, denoting $1_{\mathbb{I}}(x)=1$ if $x \in \mathbb{I}$ and $1_{\mathbb{I}}(x)=0$ if $x \notin \mathbb{I}$, Eq. (27) is satisfied with, for all $(x, \xi, s) \in \Omega \times \Omega \times \mathbb{C}_{0}^{+}$,

$$
G(x, \xi, s)=-1_{[0, x]}(\xi) \frac{\mathbf{E}_{12}(\xi-x, s)}{1+\beta s}+1_{[0,1]}(\xi) \mathbf{E}_{12}(x, s) F(\xi, s),
$$

which proves to be also defined by Eq. (28).

\subsection{Model (M1): projection of the Volterra kernels on the modal basis}

Projecting Eq. (22) on the spatial modes $e_{k}$ yields:

$$
\left\langle\left[\Gamma\left(\widehat{s_{1: n}}\right)\right]^{2} H_{n}^{(x)}\left(s_{1: n}\right)-\frac{\partial^{2} H_{n}^{(x)}\left(s_{1: n}\right)}{\partial x^{2}}, e_{k}\right\rangle=\left\langle\frac{E_{n}^{(x)}\left(s_{1: n}\right)}{1+\beta \widehat{s_{1: n}}}, e_{k}\right\rangle
$$

where $E_{n}^{(x)}$ is defined in Eqs. (23) and (24). Expanding the first member of Eq. (101) and using the linearity of the scalar product yield 


$$
\begin{aligned}
& \left\langle\left[\Gamma\left(\widehat{s_{1: n}}\right)\right]^{2} H_{n}^{(x)}\left(s_{1: n}\right), e_{k}\right\rangle-\left\langle\frac{\partial^{2} H_{n}^{(x)}\left(s_{1: n}\right)}{\partial x^{2}}, e_{k}\right\rangle \\
& =\left[\Gamma\left(\widehat{s_{1: n}}\right)\right]^{2}\left\langle H_{n}^{(x)}\left(s_{1: n}\right), e_{k}\right\rangle-\left\langle H_{n}^{(x)}\left(s_{1: n}\right), \frac{\partial^{2} e_{k}}{\partial x^{2}}\right\rangle \\
& =\left[\Gamma\left(\widehat{s_{1: n}}\right)\right]^{2} H_{n}^{[k]}\left(s_{1: n}\right)+(k \pi)^{2}\left\langle H_{n}^{(x)}\left(s_{1: n}\right), e_{k}\right\rangle \\
& =\left(\left[\Gamma\left(\widehat{s_{1: n}}\right)\right]^{2}+(k \pi)^{2}\right) H_{n}^{[k]}\left(s_{1: n}\right)
\end{aligned}
$$

Now, the second member of Eq. $(101)$ is $\left[1+\beta \widehat{s_{1: n}}\right]^{-1}\left\langle E_{n}^{(x)}\left(s_{1: n}\right), e_{k}\right\rangle$ where $E_{n}^{[k]}\left(s_{1: n}\right)=\left\langle E_{n}^{(x)}\left(s_{1: n}\right), e_{k}\right\rangle$ is given by

$$
\begin{aligned}
& E_{1}^{[k]}\left(s_{1}\right)=\left\langle\phi, e_{k}\right\rangle, \quad \text { if } n=1, \\
& E_{n}^{[k]}\left(s_{1: n}\right)=\int_{0}^{1} E_{n}^{(x)}\left(s_{1: n}\right) e_{k}(x) \mathrm{d} x \\
& =\epsilon \int_{0}^{1}\left(\sum_{\substack{p, q, r \geq 1 \\
p+q+r=n}} \int_{0}^{1}\left[\frac{\partial H_{p}^{(\xi)}\left(s_{1: p}\right)}{\partial x} \frac{\partial H_{q}^{(\xi)}\left(s_{p+1: p+q}\right)}{\partial x}\right] \mathrm{d} \xi\right. \\
& \left.\cdot \frac{\partial^{2} H_{r}^{(x)}\left(s_{p+q+1: n}\right)}{\partial x^{2}} e_{k}(x)\right) \mathrm{d} x \\
& =-\epsilon \int_{0}^{1}\left(\sum _ { \substack { p , q , r \geq 1 \\
p + q + r = n } } \int _ { 0 } ^ { 1 } \left[\sum_{\left(\ell_{1}, \ell_{2}\right) \in\left(\mathbb{N}^{*}\right)^{2}} 2 \ell_{1} \ell_{2} \pi^{2} \cos \left(\ell_{1} \pi \xi\right) \cos \left(\ell_{2} \pi \xi\right)\right.\right. \\
& \left.\cdot H_{p}^{\left[\ell_{1}\right]}\left(s_{1: p}\right) H_{q}^{\left[\ell_{2}\right]}\left(s_{p+1: p+q}\right)\right] \mathrm{d} \xi \\
& \left.\cdot\left[\sum_{m \in \mathbb{N}^{*}} 2 m^{2} \pi^{2} H_{r}^{[m]}\left(s_{p+q+1: n}\right) \sin (m \pi x) \sin (k \pi x)\right]\right) \mathrm{d} x \\
& =-\epsilon k^{2} \pi^{4} \sum_{\substack{p, q, r \geq 1 \\
p+q+r=n}} \sum_{\ell \in \mathbb{N}^{*}}\left[\ell^{2} H_{p}^{[\ell]}\left(s_{1: p}\right) H_{q}^{[\ell]}\left(s_{p+1: p+q}\right)\right] H_{r}^{[k]}\left(s_{p+q+1: n}\right), \\
& \text { if } n \geq 2 \text {. }
\end{aligned}
$$

Finally, Eqs. (101) to (105) lead to Eqs. (31) to (33) with the definition Eq. (34).

\subsection{Proof of theorem 1}

From remark 5, the kernels $H_{n}^{(x)}$ with even orders $n$ are null so that Eq.(43) is naturally satisfied from Eq. (41) in definition 1.

For odd orders, the proof is performed by induction, as follows.

For $n=1$, Eq. (44) is straightforwardly deduced by identification on Eq. (35). 
For $n \geq 3$, assume that Eqs. (43) to (45) are satisfied for all orders strictly lower than $n$. Then, from Eq. (24) and under the standard hypotheses of the Lebesgue's dominated convergence theorem (see e.g. [28]) and the Leibniz integral rule (see e.g. [25, (3.3.7)]),

$$
\begin{aligned}
E_{n}^{(x)}\left(s_{1, n}\right)=\epsilon \sum_{\substack{p, q, r \geq 1 \\
p+q+r=n}} \int_{0}^{1}\left(\left[\sum_{\mathfrak{b} \in \mathbb{A}_{p}} H_{\mathfrak{b}}\left(s_{1: p}\right) \frac{\partial e_{\mathfrak{k}}(\mathfrak{b})}{\partial x}(x)\right]\right. \\
\left.\cdot\left[\sum_{\mathfrak{c} \in \mathbb{A}_{q}} H_{\mathfrak{c}}\left(s_{p+1: p+q}\right) \frac{\partial e_{\mathfrak{k}(\mathfrak{c})}}{\partial x}(x)\right]\right) \mathrm{d} x \\
\cdot\left[\sum_{\mathfrak{d} \in \mathbb{A}_{n}} H_{\mathfrak{d}}\left(s_{p+q+1: n}\right) \frac{\partial^{2} e_{\mathfrak{k}(\mathfrak{d})}}{\partial x^{2}}(x)\right] \\
=\epsilon \sum_{\substack{p, q, r \geq 1 \\
p+q+r=n}} \sum_{(\mathfrak{b}, \mathfrak{c}, \mathfrak{o}) \in \mathbb{A}_{p} \times \mathbb{A}_{q} \times \mathbb{A}_{r}} H_{\mathfrak{b}}\left(s_{1: p}\right) H_{\mathfrak{c}}\left(s_{p+1: p+q}\right) H_{\mathfrak{d}}\left(s_{p+q+1: n}\right) \mathcal{K}_{\mathfrak{b}, \mathfrak{c}, \mathfrak{o}}(x)(106)
\end{aligned}
$$

where

$$
\begin{aligned}
\mathcal{K}_{\mathfrak{b}, \mathfrak{c}, \mathfrak{d}}(x) & =\left(\int_{0}^{1} \frac{\partial e_{\mathfrak{k}(\mathfrak{b})}}{\partial x}(x) \cdot \frac{\partial e_{\mathfrak{k}(\mathfrak{b})}}{\partial x}(x) \mathrm{d} x\right) \frac{\partial^{2} e_{\mathfrak{k}(\mathfrak{d})}}{\partial x^{2}}(x) \\
& =-\left(\pi^{2} \mathfrak{k}(\mathfrak{b}) \mathfrak{k}(\mathfrak{d})\right)^{2}\left(\delta_{\mathfrak{k}(\mathfrak{b}), \mathfrak{k}(\mathfrak{c})}\right) e_{\mathfrak{k}(\mathfrak{d})}(x) .
\end{aligned}
$$

Now, from definitions Eqs. (41) and (42), it follows that

$$
\sum_{\substack{p, q, r \geq 1 \\ p+q+r=n}} \sum_{\substack{(\mathfrak{b}, \mathfrak{c}, \mathfrak{d}) \in \mathbb{A}_{p} \times \mathbb{A}_{q} \times \mathbb{A}_{r}}} \equiv \sum_{\mathfrak{a} \in \mathbb{A}_{n}}
$$

with $\mathfrak{b}=\mathfrak{a}_{1}, \mathfrak{c}=\mathfrak{a}_{2}, \mathfrak{d}=\mathfrak{a}_{3}, p=\mathfrak{n}\left(\mathfrak{a}_{1}\right), q=\mathfrak{n}\left(\mathfrak{a}_{2}\right), r=\mathfrak{n}\left(\mathfrak{a}_{3}\right)$

Finally, from Eqs. (31) and (106) to (108), Eq. (43) is satisfied considering the definition Eq. (45) for $H_{\mathfrak{a}}$. This concludes the proof.

\subsection{State-space representation and digital implementation}

Consider a mode $e_{k}$ with $k \in \mathbb{N}^{*}$ and the associated transfer function $Q^{[k]}(s)$ defined by Eq. (34) with input $f$ and output $y$. It corresponds to a so-called second order AR-filter, which admits the following state-space representation: 


$$
\begin{aligned}
\frac{\mathrm{d} \mathbf{X}}{\mathrm{d} t}(t) & =\mathbf{A} \mathbf{X}(t)+\mathbf{B} f(t) \\
y(t) & =\mathbf{C} \mathbf{X}(t),
\end{aligned}
$$

with $\mathbf{X}=\left[\begin{array}{c}x \\ \frac{\mathrm{d} x}{\mathrm{~d} t}\end{array}\right], \mathbf{A}=\left[\begin{array}{cc}0 & 1 \\ -k^{2} \pi^{2}-\left(\alpha+\beta k^{2} \pi^{2}\right)\end{array}\right], \mathbf{B}=\left[\begin{array}{l}0 \\ 1\end{array}\right]$, and $\mathbf{C}=[1,0]$ (it can be checked that the transfer function of this system yields $C\left(s \mathbf{I}_{2}-\right.$ $\left.\mathbf{A})^{-1} \mathbf{B}=Q^{[k]}(s)\right)$. The solution $\mathbf{X}$ is given by

$$
\mathbf{X}(t)=\int_{0}^{t} \mathrm{e}^{A(t-\tau)} \mathbf{B} f(\tau) \mathrm{d} \tau+\mathrm{e}^{\mathbf{A} t} \mathbf{X}(0),
$$

so that, for all $i \in \mathbb{N}$ and $t_{i}=i T$,

$$
\mathbf{X}\left(t_{i+1}\right)=\mathrm{e}^{\mathbf{A} T} \mathbf{X}\left(t_{i}\right)+\int_{0}^{T} \mathrm{e}^{\mathbf{A}(T-\tau)} \mathbf{B} f\left(t_{i}+\tau\right) \mathrm{d} \tau .
$$

Note that for a free regime, i.e. when $f(t)=0$, Eq. (111) gives an exact resolution in the discrete-time domain: eigen-frequencies and dampings are exactly preserved. Here, to obtain a numerical simulation, we choose to approximate the input $f$ by a piecewise-linear model $\hat{f}(t) \approx f\left(t_{i}\right)+\frac{t-t_{i}}{T}\left[f\left(t_{i+1}\right)-f\left(t_{i}\right)\right]$ so that Eq. (111) becomes

$$
\mathbf{X}\left(t_{i+1}\right)=\mathrm{e}^{\mathbf{A} T} \mathbf{X}\left(t_{i}\right)+\mathbf{B}_{1} f\left(t_{i}\right)+\mathbf{B}_{0} f\left(t_{i+1}\right),
$$

with $\mathbf{B}_{1}=T^{-1} \mathbf{A}^{-2}\left[\mathbf{I}_{2}-\left(\mathbf{I}_{2}-T \mathbf{A}\right) \mathrm{e}^{\mathbf{A} T}\right] \mathbf{B}$ and $\mathbf{B}_{0}=T^{-1} \mathbf{A}^{-2}\left[-\left(\mathbf{I}_{2}+T \mathbf{A}\right)+\right.$ $\left.\mathrm{e}^{\mathbf{A} T}\right]$ B. Now, from Eq. (112),

$$
\begin{aligned}
y\left(t_{i+2}\right)= & \mathbf{C}\left(\mathrm{e}^{\mathbf{A} T}\left(\mathrm{e}^{\mathbf{A} T} \mathbf{X}\left(t_{i}\right)+\mathbf{B}_{1} u\left(t_{i}\right)+\mathbf{B}_{0} u\left(t_{i+1}\right)\right)\right. \\
& +\mathbf{B}_{1} u\left(t_{i+1}+\mathbf{B}_{0} u\left(t_{i+2}\right)\right) \\
{\left[\begin{array}{c}
y\left(t_{i+1}\right) \\
y\left(t_{i}\right)
\end{array}\right]=} & \underbrace{\left[\begin{array}{c}
\mathbf{C} \mathrm{e}^{\mathbf{A} T} \\
\mathbf{C}
\end{array}\right]}_{\mathbf{K} \text { (invertible) }} \mathbf{X}\left(t_{i}\right)+\left[\begin{array}{c}
\mathbf{C B}_{1} \\
0
\end{array}\right] u\left(t_{i}\right)+\left[\begin{array}{c}
\mathbf{C B}_{0} \\
0
\end{array}\right] u\left(t_{i+1}\right) .
\end{aligned}
$$

Then, isolating $\mathbf{X}\left(t_{i}\right)$ in Eq. (114) and substituting this solution Eq. (113) yields the recursive (scalar) equation

$$
y\left(t_{i+2}\right)=a_{1} y\left(t_{i+1}\right)+a_{2} y\left(t_{i}\right)+b_{0} f\left(t_{i+2}\right)+b_{1} f\left(t_{i+1}\right)+b_{2} f\left(t_{i}\right),
$$

with computable coefficients $\left(a_{1}, a_{2}, b_{0}, b_{1}, b_{2}\right)$. This equation is used for the simulation: it requires $N_{q}^{+}=4$ sums and $N_{q}^{\times}=5$ products, at each step. 


\section{References}

[1] S. Bilbao and J.O. Smith III. Energy-conserving difference schemes for nonlinear strings. Acta Acustica united with Acustica, pages 299-311, 2005.

[2] C. Touzé. Analyse et modélisation de signaux acoustiques et vibratoires chaotiques. Application aux instruments de percussion non-linéaires. $\mathrm{PhD}$ thesis, ENST, 2000.

[3] S.W. Shaw and C. Pierre. Normal modes of vibration for non-linear continuous systems. Journal of Sound and Vibration, 169:319-347, 1994.

[4] M.S. Nakhla and J. Vlach. A piecewise harmonic balance technique for determination of periodic response of nonlinear systems. IEEE Transactions on Circuits and Systems, 23:85-91, 1976.

[5] L. Menguy and J. Gilbert. Weakly non-linear gas oscillation in air-filled tubes: solutions and experiment. Acta Acustica, 86:798-810, 2000.

[6] Th. Hélie and M. Hasler. Volterra series for solving weakly non-linear partial differential equations: application to a dissipative burger's equation. International Journal of Control, 77(12):1071-1082, 2004.

[7] Th. Hélie. Résolution d'une équation des ondes faiblement non-linéaire par les séries de Volterra et décomposition modale. In Congrès Français d'Acoustique, Tours, 2006.

[8] J. Le Rond d'Alembert. Recherches sur la courbe que forme une corde tendue mise en vibration. Mémoires de l'académie des sciences de Berlin, pages 214$219,1747$.

[9] L. Euler. Sur la vibration des cordes. Mémoires de l'académie des sciences de Berlin, pages 69-85, 1750.

[10] C. Truesdell. Outline of the history of flexible or elastic bodies to 1788. Journal of the Acoustical Society of America, 32(12):1647-1656, 1960.

[11] G. Kirchhoff. Vorlesungen über Mathematische Physik: Mechanik. Leipzig: Teubner, 1877.

[12] G. F. Carrier. On the non-linear vibration problem of the elastic string. Quarterly of Applied Mathematics, 3:157-165, 1945.

[13] G.V. Anand. Large-amplitude damped free vibration of a stretched string. Journal of the Acoustical Society of America, 45(5):1089-1096, 1969.

[14] R. Narasimha. Non-linear vibration of an elastic string. Journal of Sound and Vibration, 8, 134-146 1968.

[15] A. Watzky. Non-linear three-dimensional large-amplitude damped free vibration of a stiff elastic stretched string. Journal of Sound and Vibration, 153:125-142, 1992. 
[16] C. Valette and Ch. Cuesta. Mécanique de la corde vibrante. Hermès, 1993.

[17] A. Chaigne and V. Doutaut. Numerical simulations of xylophones. i. timedomain modeling of the vibrating bars. Journal of the Acoustical Society of America, 101:539-557, 1997.

[18] G.C. Gorain and S.K. Bose. Uniform stability of damped nonlinear vibrations of an elastic string. Proceedings of the Indian Academy of Sciences (Mathematical Sciences), 113(4):443-449, November 2003.

[19] M. F. Ashby. Materials Selection In Mechanical Design. ButterworthHeinemann, 2004.

[20] Source for materials information. http://www matweb.com.

[21] S.P. Boyd. Volterra series: Engineering fundamentals. PhD thesis, Harvard University, 1985.

[22] M. Hasler. Systèmes non linéaires. EPFL Lausanne, 1999.

[23] W. J. Rugh. Nonlinear system theory. Web Version, 2002.

[24] F. Lamnabhi-Lagarrigue. Analyse des systèmes non linéaires. Hermès, 1994.

[25] M. Abramowitz and I.A. Stegun. Handbook of mathematical functions. New York: Dover, 1970.

[26] S. Boyd and L.O. Chua. Fading memory and the problem of approximating nonlinear operators with Volterra series. IEEE Trans. Circuits Systems, $32: 1150-1161,1985$.

[27] Th. Hélie and B. Laroche. On the convergence of Volterra series of finite dimensional quadratic MIMO systems. International Journal of Control, (to appear).

[28] Browder. Mathematical Analysis: An introduction. New York: Springer-Verlag, 1996.

[29] H. Brézis. Analyse fonctionnelle, théorie et applications. Dunod, 1999.

[30] Th. Hélie and D. Matignon. Damping models for the sound synthesis of barlike instruments. In 7th int. conf. on Systemics, Cybernetics and Informatics, Orlando, Florida, pages 541-546, July 2001.

[31] D. Roze. Simulation d'une corde avec fortes déformations par les séries de Volterra. Master's thesis, Université Pierre et Marie Curie, Paris 6, 2006.

[32] L. R. Rabiner and C. M. Rader, editors. Digital signal Processing. IEEE Press, Inc. New York, 1972.

[33] A. de Cheveigné and H. Kawahara. Yin, a fundamental frequency estimator for speech and music. Journal of the Acoustical Society of America, 111:1917-1930, Avril 2002. 